\title{
Investigations of element spatial correlation in Mn-promoted Co-based Fischer-Tropsch synthesis catalysts
}

Gregory R. Johnson ${ }^{a, \ddagger}$, Sebastian Werner $^{\mathrm{a}, \neq}$, Karen C. Bustillo ${ }^{\mathrm{b}}$, Peter Ercius ${ }^{\mathrm{b}}$, Christian Kisielowski ${ }^{\mathrm{b}}$, Alexis T. Bell ${ }^{\mathrm{a}, *}$

${ }^{a}$ Department of Chemical and Biomolecular Engineering, University of California, Berkeley, CA 94720, USA

b National Center for Electron Microscopy, Molecular Foundry, Lawrence Berkeley National Laboratory, Berkeley, CA 94720, USA

Submitted to

Journal of Catalysis

November 15, 2014

${ }^{\ddagger}$ authors contributed equally to this work

*corresponding author: bell@cchem.berkeley.edu 
Keywords

Energy Dispersive X-ray Spectroscopy (EDS), Scanning Transmission Electron Microscopy (STEM), Elemental Mapping, Image Analysis, Heterogeneous Catalysis, Fischer-Tropsch Synthesis 


\begin{abstract}
Making connections between performance and structure in bimetallic catalysts requires knowledge of how the two elements are spatially associated. Elemental maps obtained by analytical TEM methods are an invaluable tool for identifying the location of different elements, but for many samples visual inspection of elemental maps is insufficient for assessing the degree of element spatial correlation. This is particularly true for beam-sensitive materials where short mapping acquisition times lead to images with high noise and low color depth. In these situations, statistical analysis of elemental maps can be used to identify spatial correlations among the elements in a sample. In this work, the relationship between catalyst performance and bimetallic spatial association was explored using Mn-promoted Cobased Fischer-Tropsch synthesis catalysts prepared by different pretreatment methods. Mn was used as a catalyst additive to suppress methane formation. Catalysts that underwent calcination before reduction produced more methane and fewer long-chain hydrocarbons than catalysts that were directly reduced. The extent to which Co and $\mathrm{Mn}$ were spatially associated was assessed using correlation metrics, colocation plots, and histograms generated using data from STEM-EDS maps. Although both catalysts yielded visually similar elemental maps, the results of statistical analysis suggested that the calcined catalyst exhibited greater spatial segregation between the $\mathrm{Co}$ and $\mathrm{Mn}$. These findings support the hypothesis that having $\mathrm{Mn}$ in close proximity to the Co is essential for the manifestation of $\mathrm{Mn}$ promotion effects in Co-based FTS catalysts.
\end{abstract}

\title{
Dedication
}

This paper is dedicated in memory of Dr. Haldor Tøpsoe, the founder of Haldor Tøpsoe A/S and a leader in the development of new catalytic technologies. Throughout his career, Dr. Tøpsoe affirmed that an understanding of catalyst composition and structure was essential for the development of superior catalysts. The technique that particularly interested him was transmission electron microscopy because it enabled direct visualization of catalyst nanoparticles and the changes that they experience upon being used to promote chemical reactions. It is, therefore, appropriate that this article, which describes advanced TEM methods developed to provide a quantitative measure of the association of promoting elements with the element that are responsible for catalyst activity, is included in this special volume. 


\section{Introduction}

Transmission electron microscopy (TEM) is a valuable tool for characterizing catalyst particles and has been widely applied in the field of heterogeneous catalysis to assess nanoparticle size and structure. This has been demonstrated for numerous systems containing $\mathrm{Pt}, \mathrm{Au}$, or other heavy transition metals [1] [2] [3]. However, for lighter transition metals such as $\mathrm{Co}$ and $\mathrm{Cu}$, standard techniques such as brightfield TEM become more challenging due to the lower contrast of these elements against typical catalyst support materials such as silica, alumina, or titania. Scanning transmission electron microscopy with high-angle annular dark-field imaging (HAADF-STEM) produces images with contrast based on the atomic number of the material (Z-contrast), but this technique is of limited utility when investigating samples containing elements with similar atomic numbers, overlapping structures in projection, and uneven thickness [4]. For many catalyst systems, analytical electron microscopy methods such as energy dispersive spectroscopy (EDS) or electron energy-loss spectroscopy (EELS), are the only viable techniques for obtaining element-specific images with nanoscale resolution.

Acquiring elemental maps on particles or structures that are less than $10 \mathrm{~nm}$ in size means that the measured signal will be small. Particularly in EDS mapping, where X-ray generation by core-electron relaxation after excitation with the incident beam is a low probability event, it is necessary to use more beam current than is typical for imaging or to use increased acquisition times for signal averaging [5]. However, small particles are often beam-sensitive, and the integrity of the map necessitates that the incident electrons do not impart enough energy to rearrange the material being mapped. The challenge then is to balance sample damage (requiring lower beam current or total dose) and spatial resolution with the collection of enough X-ray counts to make quantitative conclusions (requiring higher beam current or total dose). Even with proper optimization of map acquisition parameters, additional challenges exist pertaining to the interpretation of map data and the determination of how associated or segregated multiple elements are with respect to each other.

Often, the assessment of element spatial association relies upon visual inspection to identify structures of different compositions in the elemental maps [6] [7] [8]. However, this approach is insufficient when interpretable motifs do not exist or when the length scale of composition heterogeneity is similar to that of the map resolution. Moreover, comparisons among samples are difficult to make if the degree of element segregation varies throughout different regions of an individual sample. Statistical tools for analyzing image similarity represent a useful alternative approach, and there are abundant examples of these tools being applied toward microscopy research. Microbiologists have long made use of various 
correlation coefficients to quantify colocation of fluorescently labeled proteins within cells [9]. Using elemental maps obtained by energy-filtered TEM, Grogger et al. were able to infer the existence of various chemical phases in alloys and ceramics through multivariate histograms [10] [11]. More recently, Parish and Brewer explored the application of principle component analysis toward STEM-EDS maps to count and identify distinct phases in ceramics [12]. Still, there has not been much evaluation of how statistical methods can be used to evaluate element segregation in samples for which sensitivity to beam damage is a prevailing concern.

These considerations about data interpretation are frequently present when studying industrially relevant catalysts, which often consist of a catalytically active metal and one or more catalytically inactive elements, referred to as promoters, that serve to increase the activity, selectivity, or stability of the active element [13]. For example, Co metal is known to be an active catalyst for Fischer-Tropsch synthesis (FTS) of hydrocarbons from $\mathrm{CO}$ and $\mathrm{H}_{2}$, and transition metal oxide promoters are frequently employed to improve product selectivity [14]. While several studies have shown that the addition of $\mathrm{Mn}$ limits the undesired formation of methane and enhances the formation of long-chain hydrocarbons used in diesel fuel, the means by which Mn affects the catalytic properties of Co are not well understood [15] [16]. Boffa et al. and Sachtler et al. have hypothesized that adsorbed CO can interact simultaneously with Co metal sites and nearby $\mathrm{Mn}$ cations so as to weaken the carbonyl bond and facilitate CO dissociation [17] [18]. If such a scheme were true, then it would imply that Mn oxide must be in contact with Co metal nanoparticles. Testing such a hypothesis requires analytical electron microscopy in order to understand the degree of spatial association between $\mathrm{Co}$ and $\mathrm{Mn}$ within these catalysts. Morales et al. have used STEM-EELS imaging to show that $\mathrm{Mn}$ locates preferentially close to $\mathrm{Co}$ in $\mathrm{TiO}_{2}$-supported Co-Mn catalysts; however, these conclusions came from visual inspection of a small number of nanoparticles [19]. There are, therefore, compelling reasons for developing ways to avoid the risks of basing conclusions on non-representative images and subjective interpretations. There are compelling motivations for developing ways to avoid the risks of basing conclusions on non-representative images and subjective interpretations. In this work, we adapt existing methods and introduce new approaches for statistically evaluating the colocation of two elements from a set of EDS maps. We demonstrate that these methods can be used even when the total number of X-ray counts per pixel is low and where binning pixels would sacrifice too much spatial resolution. These methods are then used to analyze a Mn-promoted Co catalyst to show how changes in the pretreatment of this catalyst affects the spatial relationship of $\mathrm{Mn}$ and $\mathrm{Co}$, which correlates with changes in catalyst product selectivity. 


\section{Experimental}

\subsection{TEM Sample Preparation}

Catalyst precursors were prepared by impregnating porous silica (PQ Corporation, CS-2129) with an aqueous solution of $\mathrm{Co}\left(\mathrm{NO}_{3}\right)_{2} \cdot 6 \mathrm{H}_{2} \mathrm{O}$ and $\mathrm{Mn}\left(\mathrm{CH}_{3} \mathrm{COO}\right)_{2} \cdot 4 \mathrm{H}_{2} \mathrm{O}$ (Sigma-Aldrich, $99.999 \%$ purity) followed by drying at room temperature overnight. The dried catalyst precursors were then calcined in flowing air (Praxair, zero grade) at $673 \mathrm{~K}$ or reduced in flowing $\mathrm{H}_{2}$ (Praxair, 99.999\% purity) at $673 \mathrm{~K}$ for $2 \mathrm{hr}$ with 4 $\mathrm{K} /$ min temperature ramps followed by passivation with 500 ppm $\mathrm{O}_{2}$ in He (Praxair, 99.999\% purity) at room temperature for $30 \mathrm{~min}$. In this work, catalysts prepared by these two pretreatment methods are referred to as initially calcined and directly reduced catalysts, respectively. Co weight loading was kept constant at $10 \mathrm{wt} \%$ Co in all samples; Mn loading was varied to yield samples with $\mathrm{Mn} / \mathrm{Co}$ atomic ratios of $0,0.1$, and 0.5 . The bulk elemental compositions of all catalyst samples were verified by ICP-OES (Galbraith Laboratories).

TEM samples of the catalysts were prepared by grinding $5 \mathrm{mg}$ of catalyst in a mortar for $30 \mathrm{~s}$. The ground catalyst particles were then suspended in $1 \mathrm{ml}$ of anhydrous hexane and ultrasonicated for 1 min. From this suspension, $5 \mu \mathrm{l}$ were drop-cast onto an ultra-thin carbon film with lacey carbon support CU TEM grid (Ted Pella). To remove solvent, the sample grid was dried in a vacuum oven at $373 \mathrm{~K}$ at less than 0.1 bar for $1 \mathrm{hr}$.

\subsection{TEM and STEM Imaging}

Bright-field TEM images of the Co-Mn catalysts were acquired to assess particle size statistics using an FEI Tecnai T12 microscope at the Electron Microscopy Lab at UC Berkeley operated with an accelerating voltage of $120 \mathrm{kV}$. For each catalyst sample, approximately 300 nanoparticles were used to generate particle size distributions. The surface mean diameters, also known as the Sauter mean diameters, of the nanoparticles were calculated according to Eq. 1 , where $n_{i}$ is the number of particles with diameter $d_{i}$ in a nanoparticle sample of size $N$ [20]:

$\bar{d}=\frac{\sum_{i}^{N} n_{i} d_{i}^{3}}{\sum_{i}^{N} n_{i} d_{i}^{2}}$

More detailed imaging of the metal nanoparticles within the Co-Mn catalysts was done in STEM mode using an FEI Tecnai T20 microscope and an aberration-corrected FEI Titan 80-300 (TEAM I) at the Molecular Foundry at the Lawrence Berkeley National Laboratory. On both microscopes, images were collected using an accelerating voltage of $80 \mathrm{kV}$. 


\subsection{STEM-EDS Mapping}

Hyperspectral elemental maps were recorded using a FEI Titan electron microscope equipped with a 4 segment silicon drift detector with a 0.7 steradian solid angle at the Molecular Foundry at the Lawrence Berkeley National Laboratory. Images were acquired in scanning transmission electron microscopy (STEM) mode with a probe convergence semi-angle $(\alpha)$ of $10 \mathrm{mrad}$. High-angle annular dark-field (HAADF) images were acquired with inner semi-angles $(\beta)$ greater than $70 \mathrm{mrad}$. The $X$-ray spectrum at each pixel was recorded from 0-10 keV with an energy resolution of $140 \mathrm{eV}$ and a dispersion of $10 \mathrm{eV}$ per channel. For each map, close to 100 frames were collected using active drift compensation. The mapping was performed on regions near the edges of the silica support material that were thinner than $100 \mathrm{~nm}$ so as to avoid overlapping nanoparticles, which is necessary for proper determination of spatial correlation of elements. Optimization of mapping conditions was conducted to improve X-ray counts and minimize beam damage to the sample using the following parameter ranges: 80, 200, and $300 \mathrm{kV}$ at beam currents of 0.1 to $1.0 \mathrm{nA}$ with pixel dwell times between 50 and $150 \mu \mathrm{s}$. HAADF images were acquired during EDS mapping to monitor beam damage to the sample.

Bruker Esprit software was used to process and quantify the EDS maps using the following approach. First, a fit of the Bremsstrahlung background X-rays was subtracted from the hyperspectral images using pre-defined fitting regions where no characteristic X-ray peaks appeared. Corrections for escape peaks, pile-up peaks, shelf, tail, and shift errors were applied. Contributions of X-rays from Fe and $\mathrm{Cu}$ to the $\mathrm{Mn}$ and Co peaks, which could come from the microscope column and Cu sample grids, were negligible. As an extra precaution, a peak deconvolution routine was used to remove any contribution from these elements from the spectra. Due to the high transparency of the sample to $\mathrm{Cu}$ and $\mathrm{Fe} \mathrm{K}_{\alpha} \mathrm{X}$-rays, contributions to the $\mathrm{Co}$ and $\mathrm{Mn} \mathrm{K}_{\alpha}$ peaks due to secondary fluorescence were assumed to be negligible. Elemental compositions were determined by integrating the $\mathrm{Co}_{\alpha}$ and $\mathrm{K}_{\beta}$ peaks at $6.93 \mathrm{keV}$ and $7.6 \mathrm{keV}$ and the $\mathrm{Mn} \mathrm{K}_{\alpha}$ and $\mathrm{K}_{\beta}$ peaks at $5.89 \mathrm{keV}$ and $6.49 \mathrm{keV}$; Cliff-Lorimer factors were used to calculate percent compositions. $\mathrm{Si}$ was treated as the compound $\mathrm{SiO}_{2}$ in these calculations to improve quantification accuracy.

Spectrum images using electron energy-loss spectroscopy (EELS) were acquired at 200kV on a FEI Tecnai F20. Although EELS data can often produce better spatial resolution with thin samples, the high energy of the $\mathrm{K}$ edges of $\mathrm{Co}$ and $\mathrm{Mn}$ and the lower scattering cross-section for the $\mathrm{L}$ edges produced inferior information as compared with the EDS spectrum images. 


\subsection{Data Analysis}

Statistical analysis of element spatial association was performed using custom-written software, which was released as an open-source package called QMapTools [21]. First, X-ray count maps from which the background had been subtracted and detector effects removed, as described previously, were exported from the Bruker Esprit software. This was done separately for the Co and Mn channels with the data being exported as color-coded images with the convention of red representing $\mathrm{Mn}$ and green representing Co where pixel intensity scaled linearly with X-ray counts for the respective element. To improve the statistical significance of the correlation analysis, multiple images from different regions of the same sample were used. A variety of correlation metrics, scalar values that express the level of similarity between two numerical series, were then computed using the intensity maps. For all four of the correlation metrics described below, possible values are decimal numbers ranging from -1 to 1 , where 1 represents perfect positive correlation, 0 indicates no correlation, and -1 indicates perfect negative correlation between the two elements.

The Pearson product-moment correlation coefficient (PCC), which characterizes the linearity of the relationship between two variables, was calculated according to Eq. (2) [22]. Here, $N$ is the total number of pixels in the combined elemental map; $r_{i}$ and $g_{i}$ are the intensities of the $i^{\text {th }}$ pixel for the $\mathrm{Mn}$ and Co channels, respectively; $\bar{r}$ and $\bar{g}$ are the mean pixel intensities of the $\mathrm{Mn}$ and Co channels, respectively; and $s_{r}$ and $s_{g}$ are the sample standard deviations of the $\mathrm{Mn}$ and Co channels, respectively. By normalizing using the mean intensity, differences in brightness between the two channels are controlled for, and the division by the standard deviations renders the metric insensitive to differences in image contrast [23].

$P C C=\frac{1}{(N-1)} \sum_{i=1}^{N}\left(\frac{r_{i}-\bar{r}}{s_{r}}\right)\left(\frac{g_{i}-\bar{g}}{s_{g}}\right)$

If $\mathrm{Mn}$ were to form a shell-like layer over the Co nanoparticles, then the X-ray counts for the two metals would not scale linearly together. Since the PCC tests for a linear relationship between the concentrations of the two metals, the PCC may fail to properly account for spatial association other than alloy formation. For this reason, the Spearman rank correlation coefficient (SRCC), which tests for monotonicity between the Co and Mn pixels intensities, was also computed. The formula for the SRCC is given in Eq. (3) [24]. In this equation, $R_{i}$ and $G_{i}$ are the ordinal ranks of the $\mathrm{Mn}$ and Co pixel intensities at the $i^{\text {th }}$ pixel. 
$S R C C=1-\frac{6 \sum_{i=1}^{N}\left(R_{i}-G_{i}\right)^{2}}{N\left(N^{2}-1\right)}$

Within the field of life sciences, a commonly used metric to assess colocation of fluorescent dyes using confocal microscopy is the Manders colocalization coefficient (MCC) [25]. This metric considers the fraction of pixels, weighted by intensity, containing one species that also contain the other species. For maps suffering from shot noise or low color depth, the magnitude of the signal intensities at a given location in the map may be quite unrelated to the actual element concentrations at that position. By making an assessment of element co-presence irrespective of relative channel intensities, the MCC should be relatively insensitive to these problems. In the context of this work, the fraction of Cocontaining pixels that also contained $\mathrm{Mn}$ was calculated as demonstrated in the equation below:

MCC $=\frac{\sum_{i}^{N} g_{i, \text { coloc }}}{\sum_{i}^{N} g_{i}}$ where $g_{i, \text { coloc }}=g_{i}$ if $r_{i}>0$, and $g_{i, \text { coloc }}=0$ otherwise

A limitation of these correlation coefficients is that similarity between adjacent pixels is neglected in the calculations. Since this information has been assumed to be a key component of human visual perception, Wang et al. developed the structural similarity index method (SSIM) to quantify the similarity between two images using structural information [26]. Here, the similarity of a small window of pixels is compared on both channels, and this calculation is repeated at every position in the image to generate a local SSIM value at each pixel. These values are then averaged to yield a scalar value expressing the similarity of the two images. By basing the metric on the comparison of groups of contiguous pixels, more weight should be given toward spatial correlation between the elements on length scales larger than the map resolution using the SSIM than with the other correlation metrics. The calculation of this metric was implemented using an $8 \times 8$ pixel window, a 1.5 pixel standard deviation for the Gaussian distribution applied to the window, and the same stabilizing constants as used by Wang et al.

Although the correlation metrics are relatively straightforward to interpret, information about how correlation scales as a function of channel intensities is not retained in the resulting scalar value. To investigate how much $\mathrm{Mn}$ was present where Co was least and most concentrated, plots showing the fractional Mn colocation versus normalized Co pixel intensity, referred to as colocation plots in this work, were constructed. First, the $\mathrm{Mn}$ and Co channels were divided by the highest $\mathrm{Mn}$ and Co pixel intensities in the map to yield a normalized map in which all pixel values on both channels ranged from 0 to 1 . All pixels with a given normalized Co pixel intensity, $g$, were then grouped into a set of size $N_{g}$. The 
normalized $\mathrm{Mn}$ intensities at each pixel in this set, $r_{i} / r_{\text {max }}$, were then averaged according to Eq. 5 to yield the fractional colocation of $\mathrm{Mn}$ for all pixels with a given Co intensity, $f_{M n, g}$.

$f_{M n, g}=\frac{1}{N_{g}} \sum_{i}^{N_{g}} \frac{r_{i}}{r_{\max }}$

Two-dimensional histograms of $\mathrm{Co}$ and $\mathrm{Mn}$ pixel intensities were prepared by linearly scaling the pixel intensities in each channel separately so that the mean intensities were rescaled to be 0.5 for both channels. A scatterplot for scaled $\mathrm{Mn}$ versus scaled Co intensities was then created where each data point represented one pixel in the elemental map. The scatterplot was divided into 20 bins in both dimensions and the number of data points in each bin counted. These data were then used to make a heat map equivalent to a two-dimensional histogram where the number of pixels having a given pair of scaled Co and $\mathrm{Mn}$ intensities was represented by a color scale. The initial normalization placed images with different total X-ray counts onto a common scale and located the centroid of the histogram at the center of the plot. Scaling the $\mathrm{Mn}$ and $\mathrm{Co}$ in this manner made any datapoints that fell along the diagonal line running from the origin through the center of the plot have $\mathrm{Mn} / \mathrm{Co}$ ratios equivalent to that of the bulk catalyst. By observing how much histogram intensity was located on or away from the diagonal parity line, a quick determination of the relative extents of segregation in different samples could be made. Even though data points could exist at any positive number along both axes of the histogram, the plotted axes were truncated at 1 because the number of data points with either scaled $\mathrm{Mn}$ or Co intensities above 1 was negligible for both the maps of the Co-Mn catalysts and those based on the simulated images.

Additionally, histograms of atomic composition on the basis of Co and Mn were constructed. These plots were prepared by calculating the relative concentrations of $\mathrm{Co}$ and $\mathrm{Mn}$ at each pixel using the CliffLorimer method with $4 \times 4$ pixel binning to increase accuracy [27]. Cliff-Lorimer factors for compositions in terms of atomic percent at the appropriate accelerating voltage were obtained from the Bruker Esprit software.

\subsection{Catalytic Testing}

Experiments to determine catalyst activity and selectivity were conducted using a fixed-bed microreactor. In a typical experiment, approximately $50 \mathrm{mg}$ of passivated catalyst diluted with $100 \mathrm{mg}$ of silica were mixed together and loaded into a quartz glass reactor. The reactor was then heated at 4 $\mathrm{K} / \mathrm{min}$ from room temperature to $673 \mathrm{~K}$ in $100 \mathrm{ml} / \mathrm{min}_{2}$ at 1 bar to reduce the catalyst. After holding at 
$673 \mathrm{~K}$ for $120 \mathrm{~min}$, the reactor was cooled to $493 \mathrm{~K}$ and the inlet feed was switched to syngas with $\mathrm{H}_{2} / \mathrm{Co}$ $=2$ (Praxair, $\mathrm{CO} 99.9 \%$ purity and $\mathrm{H}_{2}$ 99.999\% purity) to start the Fischer-Tropsch reaction. Measurements were done at steady state at various CO conversions by manipulating the inlet feed volumetric flow rate. The chemical composition of the reactor outlet was determined using an Agilent 6890 gas chromatograph with FID and TCD detectors to measure hydrocarbons and CO, respectively.

\section{Results and Discussion}

\subsection{TEM and HRSTEM Imaging}

TEM images of the Co-Mn catalysts were collected to assess the size distribution and structure of the metal nanoparticles supported on silica. Typical bright field TEM images for an unpromoted and Mnpromoted catalyst $(\mathrm{Mn} / \mathrm{Co}=0.1)$ are presented in Fig. 1 . Although the metal nanoparticles are visible against the silica support material, it is not possible to determine the relative abundance of the two metals within the nanoparticles owing to the similar atomic numbers of $\mathrm{Co}$ and $\mathrm{Mn}$ and the ambiguous nature of TEM contrast. The surface mean diameters of the unpromoted, $\mathrm{Mn} / \mathrm{Co}=0.1$, and $\mathrm{Mn} / \mathrm{Co}=0.5$ catalysts were calculated to be $9.4 \pm 2.1 \mathrm{~nm}, 9.1 \pm 1.8 \mathrm{~nm}$, and $8.9 \pm 2.6 \mathrm{~nm}$, respectively. No significant differences in nanoparticle morphology were observed among the three catalyst samples; most nanoparticles had near-spherical geometry.

HAADF-STEM images provided better contrast of the metal nanoparticles against the silica support as shown in Fig. 2. Although the atomic lattice of the metal nanoparticles was visible in images acquired under HRSTEM conditions (Fig. 2B), it was not possible to infer the presence of Mn using these data. Since $\mathrm{Co}$ and $\mathrm{Mn}$ nanoparticles oxidize in air, all reduced catalyst samples were passivated with dilute $\mathrm{O}_{2}$ at ambient temperature before immobilization on TEM sample grids. Various mixed oxide phases have been reported for $\mathrm{Co}$ and $\mathrm{Mn}$ so both the stoichiometry and crystal structure of the nanoparticles were indeterminate, and reliable phase identifications and calculation of lattice parameters was not possible [28].

\subsection{Acquisition of Elemental Maps by STEM-EDS}

Elemental maps obtained by STEM-EDS proved to be a robust method for locating metal nanoparticles and distinguishing between $\mathrm{Co}$ and $\mathrm{Mn}$. To optimize map acquisitions, the interplay between STEM-EDS imaging conditions, X-ray count rates, and effective map resolution was explored using reference materials and a Mn-promoted catalyst ( $\mathrm{Mn} / \mathrm{Co}=0.1)$. Supporting Information SI2 shows the effect of accelerating voltage and beam current on the X-ray count rate using a $60 \mathrm{~nm}$-thick reference $\mathrm{Cu}$ foil, 
which was estimated to be of comparable mass thickness to the imaged regions of the Co-Mn catalyst samples. X-ray count rates increased linearly with beam current and decreased with higher accelerating voltages. These observations were consistent with the phenomenon of scattering cross-sections decreasing as accelerating voltage increases [29]. In order to achieve sufficient beam current, the strength of the first condenser lens is reduced which results in a larger probe size than is typically used for high resolution STEM. Since accelerating voltage is inversely related to beam spreading, it can be inferred that effective map resolution would be poorer at higher beam currents because of the larger probe size and at lower accelerating voltages [30]. For this study, an accelerating voltage of $200 \mathrm{kV}$ and beam current of about $0.6 \mathrm{nA}$ was deemed to be an acceptable compromise between resolution and acquisition time, and most images were acquired at these conditions. Based on measurements in which oxide multilayers in a reference sample were resolved, the size of the electron probe was estimated to be $0.8 \mathrm{~nm}$. Typically, $512 \times 512$ pixel maps were acquired with $0.54 \mathrm{~nm}$ pixel length, which gave dose rates of about 50,000 e/nm ${ }^{2}$-s. The effective spatial resolution was estimated to be between 0.8 and 1.1 $\mathrm{nm}$ based on the single-scattering model [31]. Consequently, binning of the pixels was not performed, except for the composition histograms, so as not to discarded valid spatial information.

Beam damage was another factor that participated in the selection of operating parameters. It was observed that extended mapping times led to significant densification of the silica support, which placed an upper limit on elemental map acquisition times. As seen in Fig. 3C, the porous structure of the silica support had collapsed appreciably after 45 min of scanning under the electron beam at $200 \mathrm{kV}$ and 0.6 $\mathrm{nA}$. Videos of beam damage to the support using various accelerating voltages, beam currents, and pixel dwell times are included in Supporting Information SI3. At identical total electron doses to the sample, similar levels of sample restructuring were observed when varying pixel dwell times and beam currents. These observations suggest that damage to the sample was more a function of total dose rather than dose rate in the tested range of parameters. Sample damage appeared to decrease slightly at higher accelerating voltages, which was consistent with the restructuring being caused by possibly a combination of radiolysis and diffusion [29]. To prevent sample damage from substantially affecting the elemental maps, the live HAADF-STEM image was monitored so that the mapping could be terminated before significant sample restructuring occurred (Fig. 3B).

Silica particles began to move upon starting the beam rastering so inevitably there was a small amount of motion in the support during the mapping. Maps showing gross damage to the support were discarded. Of the maps that were analyzed, most features moved no more than $5 \mathrm{~nm}$ between the initial 
and final HAADF-STEM images. The use of active drift compensation, which tilts the beam to follow features of interest, was not relevant for averting the errors introduced by the motion of overlapping silica particles relative to each other. By itself, this phenomenon would limit the effective length scale at which segregation could be detected to perhaps no less than $5 \mathrm{~nm}$. No evidence for knock-on damage was observed in these samples at the tested acceleration voltages. Particle sintering was an unlikely damage mechanism because the particle size distributions visible in STEM-EDS were comparable to those obtained from BF images. Another damage-related concern is the possibility of Oswald ripening, which has been reported as a prominent mechanism for damage to nanoparticle specimens at high enough beam currents [32]. In this mechanism, atoms on the surface of small particles may become mobilized under the beam and diffuse to larger particles. By surface free energy arguments, most of the $\mathrm{Mn}$ should be located on the surface of the metal nanoparticles so Oswald ripening may affect the nanoparticle compositions without appreciable changes in the particle sizes [33]. Using very low dose rates may help avoid this type of damage [34]. However, STEM-EDS map acquisition times would be impractically long for this system. Since this type of beam-sensitivity would not be readily visible in the HAADF-STEM images owing to the difficulty of identifying metal nanoparticles against the silica support in these images, Oswald ripening may be an additional contribution to the level of spatial association in the elemental maps.

Representative elemental maps of a Mn-promoted Co catalyst $(\mathrm{Mn} / \mathrm{Co}=0.5)$ are shown in Fig. 4. Maps from multiple catalyst particles were acquired to confirm that these images were representative of the sample. The boxed region in the HAADF-STEM image (Fig. 4A) shows the region over which the elemental maps were acquired. Although metal nanoparticles are visible in the HAADF-STEM image, identifying which features belong to the nanoparticles and to the edges of the overlapping support particles is challenging. However, the EDS maps for Co and Mn (Figs. 4C and 4D, respectively) clearly reveal the locations of the nanoparticles. By comparing spatial features in both the Co and Mn maps, it is apparent that there is a certain degree of correlation between the locations of the two elements because regions of higher Co intensity appear at regions of higher $\mathrm{Mn}$ intensity. The two maps are not identical though, and $\mathrm{Mn}$ appears to have dispersed over the silica support more so than the Co, which has been speculated to occur in related Co-Mn catalysts [35]. Without a single well-defined morphology, such as alloyed or separate Co and Mn nanoparticles, it would be difficult to make comparisons between catalyst samples. Consequently, statistical methods for determining the extent of Co-Mn spatial association were explored. 


\subsection{Correlation Analyses of Simulated Elemental Maps}

To validate the ability of the statistical analysis methods to evaluate spatial correlation, simulated images of nanoparticles with various compositions and structures were generated. The maps were generated stochastically using a Poisson process to simulate detection of X-ray counts. Three levels of spatial association between $\mathrm{Co}$ and $\mathrm{Mn}$ were considered (in order of increasing element segregation): nanoparticles with alloyed $\mathrm{Co}$ and $\mathrm{Mn}$, small $\mathrm{Mn}$ nanoparticles decorating the surface of larger Co nanoparticles, and separate $\mathrm{Co}$ and $\mathrm{Mn}$ nanoparticles with uncorrelated positions. Representative images of these three test cases are shown in Fig. 5A.

Correlation metrics computed using sets of 20 simulated images for each test case are given in Table 1. Values for the PCC, SRCC, MCC, and SSIM were close to unity for the alloying case, which represented maximal spatial correlation between the two elements. Lower values for the correlation metrics were found for the surface associated case, and the segregated case had the lowest value metrics. For the latter, the MCC and SSIM were less sensitive to segregation having relatively high values of 0.27 and 0.18 , respectively, compared to the 0.03 and 0.02 of the PCC and SRCC. This would be expected for the MCC because a portion of the randomly distributed Co and Mn nanoparticles will always overlap when particle densities are sufficiently high. The SSIM may yield higher than expected estimates of similarity due to its incorporation of information about adjacency. Nevertheless, all four metrics were shown to discriminate adequately among the test images.

Fig. 5B shows colocation plots for the three test cases. In the case of alloyed nanoparticles, a slope of unity was observed because there were approximately equal intensities of Co and $\mathrm{Mn}$ at each location in the simulated elemental maps. Surface decorating Mn particles yielded a colocation plot with a smaller slope which showed a plateau at higher Co intensities. The Co nanoparticles were simulated as three dimensional spheres so the pixels with maximum $\mathrm{Mn}$ intensities, which were used to normalize the data, were likely to have occurred near the edges of the projected area formed by the Co nanoparticles. This meant that fractional Mn colocation should be less than unity where Co intensities were highest, which was at the center of the projected area formed by the Co nanoparticles. The case of uncorrelated, separate Co and $\mathrm{Mn}$ nanoparticles resulted in a plot with a flat distribution, which was the expected result if $\mathrm{Co}$ and $\mathrm{Mn}$ intensities were not correlated. For this case, the average fractional $\mathrm{Mn}$ colocation was dictated by the density of nanoparticles in the simulated map, with fractional Mn colocation values increasing as nanoparticles density increased. 
The two-dimensional histograms for $\mathrm{Co}$ and $\mathrm{Mn}$ intensities presented in Fig. $5 \mathrm{C}$ gave characteristic patterns for different levels of element segregation. Intense histogram density along the diagonal parity line for the alloyed nanoparticles was consistent with all pixels having compositions similar to that of the bulk. With increasing segregation, more histogram density was observed away from the parity line and toward the axes. For the spatially uncorrelated nanoparticles, essentially all histogram intensity fell along the axes, which indicated most pixels contained either no Mn or no Co signal. The composition histograms shown in Fig. 5D were consistent with the observed spatial distributions in the simulated maps. The alloyed nanoparticles with homogeneous composition gave a narrow, monomodal distribution. Surface decoration of the Co nanoparticles by smaller Mn nanoparticles, showed a broader composition distribution with the appearance of pixels with compositions near $0 \%$ and $100 \% \mathrm{Mn}$. The spatially uncorrelated nanoparticles showed strong signs of segregation with most histogram density close to either $0 \%$ or $100 \% \mathrm{Mn}$.

In situations where beam damage to the sample limits the permissible map acquisition times, elemental maps may have low color depth due to insufficient X-ray counts. Here, color depth refers to the number of distinct values for pixel intensities in a given channel, and an illustration of the concept is provided in Supporting Information SI4. The effect of lower color depth on the output of the statistical methods was tested using the simulated images which were modified to have lower color depth. As shown in Supporting Information SI5 for the surface associated test case, the correlation metrics were quite insensitive to this degradation in data quality, and the colocation plot was unaffected provided that the Co channel had high color depth. Fewer intensity values translated to missing data points in the colocation plot if the Co channel was modified, and the two-dimensional histograms were severely impacted when the color depth of either the Mn or Co channels were lowered because this plot displays information about the pixel intensities of both channels. These results suggest that the correlation metrics could be used for assessing element segregation in maps with low color depth. Since the colocation plot only required sufficient color depth in one channel, the plot could be an appropriate tool to use when one element is more dilute than the other.

Supporting Information SI6 shows the effects of Gaussian noise on the output of the statistical methods. With the exception of the MCC, increasing levels of noise caused the metrics to report lower levels of element spatial correlation, which was consistent with the noise being spatially uncorrelated. The PCC was least sensitive in the presence of low noise, but the SSIM demonstrated the best performance at high noise levels where the noise began to visually obscure the simulated signal from the nanoparticles. 
The slope of the colocation plots flattened as noise levels increased. Low levels of noise overwhelmed the real signal in the two-dimensional histograms owing to the greater number of pixels where no nanoparticles where located. A judicious pixel intensity cutoff or pixel mask could be used to remove the contribution of these pixels from the plot.

It must be emphasized that even though these analysis methods were tested on and could discriminate between very dissimilar structural patterns, the objective was not to identify the specific morphology of the nanoparticles. Rather, the intention was to extract a relative quantitative measure of how segregated the two elements were for the purpose of making comparisons between catalysts.

\subsection{Correlation Analysis of Co-Mn Catalysts}

STEM-EDS maps of the initially calcined and directly reduced catalysts after FTS reaction were acquired to determine how closely associated the Mn promoter was with respect to the active Co metal. Representative elemental maps for the two catalysts are presented in Fig. 6A. From visual inspection of the maps, it is clear that there are Mn-rich and Mn-poor regions, but it is difficult to establish which catalyst exhibited a greater degree of spatial segregation between the two elements. This is complicated by the apparent tendency of the two metals to spread more evenly over the silica support after exposure to syngas, shown in Fig. 6A, compared to samples that had not been exposed to syngas, represented by Fig. 4. Scanning tunneling microscopy studies have provided evidence that Co surface atoms may become mobile under typical FTS conditions leading to catalyst restructuring [36]. Moreover, Prieto et al. reported that Co nanoparticles can flatten during reaction so as to increase the area of contact between the nanoparticles and support [37]. Such restructuring would be similar to the phenomenon observed in the catalysts of this work. A more precise determination of element spatial correlation was made using the statistical methods introduced previously.

Correlation metrics for these catalysts based on a set of over 10 map images for each catalyst are presented in Table 2. The directly reduced sample had relatively high correlation values of 0.74 (PCC), 0.78 (SRCC), and 0.56 (SSIM); lower values of 0.28 (PCC), 0.34 (SRCC), and 0.26 (SSIM) were calculated for the initially calcined sample. The PCC and SRCC metrics had similar values for the directly reduced sample, which suggests that the relationship between the Co and $\mathrm{Mn}$ intensities in this sample were relatively linear. This is visually evident in the $2 \mathrm{D}$ histograms discussed later in this section in which most histogram intensity fell along the diagonal parity line. These two metrics also had similar values for the initially calcined sample, although the difference between the two metrics was slightly larger. The PCC metric probes the more stringent criterion of linearity between the Co and $\mathrm{Mn}$ intensities rather than 
monotonicity as does the SRCC, which would make the PCC respond more sensitively to higher segregation. The lower value of the PCC metric compared to the SRCC was likely due to segregation breaking the linearity of the relationship between the Co and $\mathrm{Mn}$ intensities more so than the monotonicity of the relationship. Although the SSIM metric differentiated between the two catalysts, this metric consistently gave lower values than the PCC and SRCC metrics, which was a behavior also seen with the simulated images. Making a direct comparison between the magnitude of the SSIM values and those of the other metrics for the same sample would not be informative because the SSIM is a fundamentally different calculation which aims to quantify differences in images as perceived by the human eye, whereas the PCC and SRCC only assess the relationship between the Co and Mn intensities at each pixel. In general, the metrics should be viewed as ordinal values rather than cardinal values. The magnitude of each metric increases with higher spatial correlation so comparing the same metric for different samples yields information about which sample exhibits more spatial association. However, there is no inherent meaning to the absolute value of the metric other than the limiting values of $-1,0$, and 1. The MCC values were close to unity for both catalysts due to the baseline Mn signal over much of the catalyst support. An intensity cutoff could be used to remove regions of fewer $\mathrm{Mn}$ X-ray counts from the MCC calculation, but this would be a subjective correction that could introduce biases that overstate element segregation. Without any guide for an appropriate cutoff threshold, the MCC proved to be an unsuitable measure of element spatial association in these samples. Nevertheless, the PCC, SRCC, and SSIM metrics all indicated that the initially calcined sample had a larger degree of spatial segregation between the Co and $\mathrm{Mn}$ compared to the directly reduced sample.

Colocation plots for the two Mn-promoted catalysts are included in Fig. 6B. A much steeper slope in the plot for the directly reduced compared to that for the initially calcined catalyst was observed. This can be interpreted as indicating a higher correlation between the Co and Mn concentrations in the directly reduced sample. A related aspect of these plots is the higher fractional Mn colocation at low Co pixel intensities in the plot for the initially calcined catalyst, which is consistent with there being more Mnrich pixels. Interestingly, neither plot shows high fractional Mn colocation at high Co pixel intensities, which indicates the presence of regions with high concentrations of Co without significant $\mathrm{Mn}$ copresence in both catalysts. The representative elemental maps (Fig. 6A), which both contain several bright green regions on the scale of $10-40 \mathrm{~nm}$, are consistent with this interpretation of the colocation plots. 
Two-dimensional histograms, shown in Fig. 6C, provided further evidence of greater element spatial correlation in the directly reduced sample compared to the initially calcined sample. Most of the histogram distribution for the directly reduced sample was located more closely to the diagonal parity line, which indicates that a relatively high fraction of pixels had compositions close to that of the bulk. In contrast, more of the histogram distribution for the initially calcined catalyst, which suggests there were more pixels that were enriched in either Co or $\mathrm{Mn}$. These interpretations are also evident in the atomic composition histograms for the two catalysts, which are presented in Fig. 6D. A narrower composition distribution was observed for the directly reduced catalyst. Local maxima are present in the composition histograms at $0 \%$ and $100 \% \mathrm{Mn}$. These features are due to there being pixels for which no X-ray counts were recorded at the Mn or Co peaks, respectively, which was likely a consequence of baseline noise over holes in the specimen. For both catalysts, the mean of the composition histograms was equivalent to the bulk Mn/Co ratio determined by ICP to within $5 \%$.

Taken together, the results of the correlation analyses imply that the initially calcined catalyst demonstrated greater spatial segregation between the Co and $\mathrm{Mn}$. However, it is important to recognize that beam damage to the sample could be a contribution to the observed levels of element association in the samples. Any motion of overlapping silica particles and Oswald ripening of the Co-Mn nanoparticles would likely inflate the estimates of element spatial correlation. Nevertheless, a rationalization of the greater observed segregation in the initially calcined can be made by analogy to studies of the thermal decomposition of Co precursors in silica-supported catalysts. Girardon et al. reported that the decomposition of $\mathrm{Co}\left(\mathrm{CH}_{3} \mathrm{COO}\right)_{2}$ in air led to greater formation of $\mathrm{Co}$ silicates than if $\mathrm{Co}\left(\mathrm{NO}_{3}\right)_{2}$ was used as the precursor [38]. This phenomenon was attributed to the acetate decomposition being exothermic and the nitrate decomposition being endothermic. Thermogravimetric analysis of the Co-Mn catalyst precursor in air (Supporting Information S17) confirmed that the decomposition of $\mathrm{Mn}(\mathrm{CH} 3 \mathrm{COO})_{2}$ was also exothermic, and the decomposition of $\mathrm{Mn}\left(\mathrm{CH}_{3} \mathrm{COO}\right)_{2}$ in $\mathrm{H}_{2}$ has been reported as being endothermic [39]. Compared to a direct reduction of the catalyst precursor, an oxidative pretreatment step may provide localized heat that facilitates dispersal of the Mn over the support and contributes to partial integration of the Mn into the silica.

Similar observations of more extensive bimetallic interactions after direct reduction pretreatment compared to calcination have been reported for Pt-Re catalysts based on catalytic data [40]. Higher segregation between metals due to calcination was also inferred from infrared spectroscopy of adsorbates on Pt-Ru catalysts [41]. With regard to Co-Mn catalysts, den Breejen et al. acquired 
elemental maps of Co-Mn-Pt catalysts calcined with either NO or air and found pretreatment in NO to promote higher dispersions of the both $\mathrm{Co}$ and $\mathrm{Mn}$ [42]. Other studies of Co-Mn catalysts using analytical electron microscopy techniques have been published, but data interpretation only consisted of visual inspection of a small number of nanoparticles [19] [15] [43].

\subsection{Catalytic Performance}

A comparison of the FTS product selectivities for the initially calcined and directly reduced $\mathrm{Mn}$ promoted catalysts $(\mathrm{Mn} / \mathrm{Co}=0.5)$ is presented in Fig. 7. Selectivity data for an unpromoted catalyst $(\mathrm{Mn} / \mathrm{Co}=0)$ prepared by direct reduction is included for reference. For the unpromoted catalyst, the selectivity toward methane was $33 \%$ on a molar carbon basis. The presence of $\mathrm{Mn}$ in the catalyst was found to decrease the methane selectivity, but to different extents depending on the catalyst preparation method. The Mn-promoted catalyst that was initially calcined had a methane selectivity of $26 \%$, whereas the directly reduced counterpart had a more favorable selectivity of $21 \%$. Selectivities toward $\mathrm{C}_{5+}$ hydrocarbons, a product fraction which includes all hydrocarbons containing five or more carbon atoms, followed the opposite trend. The unpromoted reference catalyst had a C5+ selectivity of $36 \%$, the initially calcined Co-Mn catalyst had a selectivity of $41 \%$, and the directly reduced Co-Mn catalyst had a selectivity of $48 \%$. An initially calcined unpromoted catalyst was found to give similar selectivities to those of the directly reduced unpromoted catalyst ( $34 \%$ methane and $34 \% \mathrm{C}_{5+}$ ). All catalysts exhibited constant carbon number product selectivities as a function of CO conversion up to conversions of $25 \%$, which was consistent with our previous findings for these catalysts when operating at atmospheric pressure [16].

Using the correlation analysis results from the elemental maps, a connection can be made between catalyst performance and the spatial association between Co and $\mathrm{Mn}$. The least methane was produced by the promoted catalyst exhibiting the highest level of spatial correlation between Co and Mn. This suggests that the selectivity promotion effects emerge from the two elements being in close proximity; specifically, the required proximity is likely to be at or smaller than the map resolution, which is what is tested by the correlation analysis. The comparable selectivities for the unpromoted catalysts prepared by initial calcination and direct reduction support the interpretation that differences in $\mathrm{Mn}$ spatial distribution are responsible for the observed selectivity differences in the promoted catalysts rather than any inherent property of the pretreatment steps on the Co. The possibility that the observed selectivity differences for the three catalysts were due to nanoparticle size effects is unlikely given that the surface mean diameters of the catalysts were similar. Moreover, FTS on Co-based catalysts has been 
shown to be insensitive to particle size above diameters of about $6 \mathrm{~nm}$, which was the case for all catalysts in this work [44].

\section{Conclusions}

As demonstrated by this investigation of Co-Mn FTS catalysts, non-analytical TEM techniques can be insufficient for distinguishing between elements when atomic numbers are similar or when nanoparticles are dispersed over irregularly structured supports. Elemental mapping can reliably identify the location of these multi-metal nanoparticles, but acquisition times long enough to generate sufficient X-ray counts for traditional quantification are often not feasible due to beam damage. Low color depth and low signal-to-noise ratios can lead to erroneous composition histograms unless significant pixel binning is used. However, such measures reduce the resolution at which variations in composition can be detected. To make comparisons about element segregation among beam-sensitive catalysts, a variety of statistical methods can be employed.

Thorough testing of the statistical methods using simulated elemental maps demonstrated that the methods could serve as quantitative descriptors of element segregation. Of the correlation metrics considered in this work, the PCC proved to be the most sensitive to changes in element segregation. However, under high noise conditions the SSIM performed the best. Colocation plots required high color depth in the map for at least one channel; two-dimensional histograms required sufficient color depth in both channels. In general, it was observed that the statistical methods that conveyed more information about the co-presence of the two elemental had more demanding data quality requirements.

These methods were used to assess metal segregation in Co-Mn FTS catalysts that had the same bulk composition, but where one catalyst was calcined in air and the other reduced in $\mathrm{H}_{2}$ during the decomposition of the Co and Mn precursors at elevated temperature. Although the elemental maps for both catalysts were visually similar, it was determined by statistical analysis that the directly reduced catalyst exhibited a greater degree of spatial association between Co and $\mathrm{Mn}$. It is possible that beaminduced damage to the sample, both by support densification and Oswald ripening of the nanoparticles, affected the observed element spatial correlation so as to limit the resolution at which element segregation was detectable. However, the elemental maps displayed composition variations at length scales larger than $20 \mathrm{~nm}$. Equivalent loadings of $\mathrm{Mn}$ promoter were added to both catalysts, but the directly reduced catalyst demonstrated the lowest methane selectivity and highest $\mathrm{C}_{5+}$ selectivity. These findings suggest that a performance-structure relationship may exist whereby higher spatial association between the Co and $\mathrm{Mn}$ is linked to improved product selectivities. This connection is consistent with 
the guiding hypothesis that $\mathrm{Co}$ and $\mathrm{Mn}$ must be in close proximity for cooperative interactions between the metals and adsorbed CO.

\section{Acknowledgments}

Funding for this work was provided by $\mathrm{BP}$ through the $X \mathrm{XC}^{2}$ program. Microscopy experiments were performed at the UC Berkeley Electron Microscopy Lab and the Molecular Foundry, Lawrence Berkeley National Lab, which is supported by the U.S. Department of Energy under Contract \# DE-AC0205CH11231. The authors acknowledge helpful discussions with Dr. Jim Ciston and Dr. Cheng-Yu Song. 


\section{References}

[1] Z. Liu, X. Y. Ling, X. Su and J. Y. Lee, "Carbon-Supported Pt and PtRu Nanoparticles as Catalysts for a Direct Methanol Fuel Cell," J. Phys. Chem. B, Vols. 8234-8240, no. 24, p. 108, 2004.

[2] A. Wolf and F. Schüth, "A systematic study of the synthesis conditions for the preparation of highly active gold catalysts," Appl. Catal. A-Gen., vol. 226, pp. 1-13, 2002.

[3] M. Ritschel, A. Leonhardt, D. Elefant, S. Oswald and B. Büchner, "Rhenium-Catalyzed Growth Carbon Nanotubes," J. Phys. Chem. C, vol. 111, pp. 8414-8417, 2007.

[4] D. O. Klenov and S. Stemmer, "Contributions to the contrast in experimental high-angle annular dark-field images," Ultramicroscopy, vol. 106, pp. 889-901, 2006.

[5] C. M. Parish and L. N. Brewer, "Key Parameters Affecting Quantitative Analysis of STEMEDS Spectrum Images," Microsc. Microanal., vol. 16, pp. 259-272, 2010.

[6] T. Zhu and M. Flytzani-Stephanopoulos, "Catalytic partial oxidation of methane to synthesis gas over Ni-CeO2," Appl. Catal. A-Gen., vol. 208, pp. 403-417, 2001.

[7] E. Sayah, D. Brouri, Y. Wu, A. Musi, Patrick Da Costa and P. Massiani, "A TEM and UV-visible study of silver reduction by ethanol in Ag-alumina catalysts," Appl. Catal. A-Gen., vol. 406, pp. 94-101, 2011.

[8] K. Xiao, Z. Bao, X. Qi, X. Wang, L. Zhong, K. Fang, M. Lin and Y. Sun, "Structural evolution of CuFe bimetallic nanoparticles for higher alcohol synthesis," J. Mol. Catal. A-Chem., vol. 378, pp. 319-325, 2013.

[9] K. W. Dunn, M. M. Kamocka and J. H. McDonald, "A practical guide to evaluating colocalization in biological microscopy," Am. J. Physiol-Cell Ph., vol. 300, pp. C723-C742, 2001.

[10] W. Grogger, F. Hofer and G. Kothleitner, "Quantitative Chemical Phase Analysis of EFTEM Elemental Maps Using Scatter Diagrams," Micron, vol. 29, no. 1, pp. 43-51, 1998.

[11] W. Grogger and K. M. Krishnan, "Scatter Diagram Analysis of Cr Segregation in Co-Cr Based Recording Media," IEEE T. Magn., vol. 37, no. 4, pp. 1465-1467, 2001.

[12] C. M. Parish and L. N. Brewer, "Multivariate statistics applications in phase analysis of STEM-EDS spectrum images," Ultramicroscopy, vol. 110, pp. 134-143, 2010.

[13] G. Jacobs, T. K. Das, Y. Zhang, J. Li, G. Racoillet and B. H. Davis, "Fischer-Tropsch synthesis: 
support, loading, and promoter effects on the reducibility of cobalt catalysts," Appl. Catal. A-Gen., vol. 233, pp. 263-281, 2002.

[14] A. Y. Khodakov, W. Chu and P. Fongarland, "Advances in the Development of Novel Cobalt Fischer-Tropsch Catalysts for Synthesis of Long-Chain Hydrocarbons and Clean Fuels," Chem. Rev., vol. 107, no. 5, pp. 1692-1744, 2007.

[15] G. Bezemer, P. Radstake, U. Falke, H. Oosterbeek, H. Kuipers, A. van Dillen and K. de Jong, "Investigation of promoter effects of manganese oxide on carbon nanofiber-supported cobalt catalysts for Fischer-Tropsch synthesis," J. Catal., vol. 237, pp. 152-161, 2006.

[16] A. Dinse, M. Aigner, M. Ulbrich, G. R. Johnson and A. T. Bell, "Effects of Mn promotion on the activity and selectivity of $\mathrm{Co} / \mathrm{SiO} 2$ for Fischer-Tropsch Synthesis," Journal of Catalysis, vol. 288, pp. 104-114, 2012.

[17] W. M. H. Sachtler, D. F. Shriver, W. B. Hollenberg and A. F. Lang, "Promoter action in Fischer-Tropsch Catalysis," J. Catal., vol. 92, no. 2, pp. 429-431, 1985.

[18] A. B. Boffa, C. Lin, A. T. Bell and G. A. Somorjai, "Lewis acidity as an explanation for oxide promotion of metals: implications of its importance and limits for catalytic reactions," Catal. Lett., vol. 27, pp. 243-249, 1994.

[19] F. Morales, D. Grandjean, F. M. F. de Groot, O. Stephan and B. M. Weckhuysen, "Combined EXAFS and STEM-EELS study of the electronic state and location of $\mathrm{Mn}$ as promoter in Cobased Fischer-Tropsch catalysts," Phys. Chem. Chem. Phys., vol. 7, pp. 568-572, 2005.

[20] A. K. Datye, Q. Xu, K. C. Kharas and J. M. McCarty, "Particle size distributions in heterogeneous catalysts: What do they tell us about the sintering mechanism?," Catal. Today, vol. 111, pp. 59-67, 2006.

[21] S. Werner and G. R. Johnson, "QMapTools," [Online]. Available: http://dx.doi.org/10.5281/zenodo.11384. [Accessed 19 2014].

[22] K. Pearson, "Mathematical Contributions to the Theory of Evolution. III. Regression, Heredity, and Panmixia," Philos. Trans. R. Soc. Lond. Ser. A, vol. 187, pp. 253-318, 1896.

[23] A. A. Goshtasby, Image Registration: Principles, Tools, and Methods, London: Springer, 2012.

[24] J. L. Myers, A. D. Well and R. F. Lorch, Jr., Research Design and Statistical Analysis, 3rd ed., New York: Routledge, 2010.

[25] E. M. M. Manders, F. J. Verbeek and J. A. Aten, "Measurement of co-localization of objects 
in dual-color confocal images," J.Microsc., vol. 169, no. 3, pp. 375-382, 1993.

[26] Z. Wang, A. C. Bovik, H. R. Sheikh and E. P. Simoncelli, "Image Quality Assessment: From Error Visibility to Structural Similarity," IEEE T. Image Process., vol. 13, no. 4, pp. 600-612, 2004.

[27] G. Cliff and G. W. Lorimer, "The quantitative analysis of thin specimens," J. Microsc., vol. 103, no. 2, pp. 203-207, 1975.

[28] A. M. Yankin and V. F. Balakirev, "Phase Equilibria in the Co-Mn-Ti-O System over Wide Ranges of Temperatures and Oxygen Pressures," Inorg. Mater., vol. 38, no. 4, pp. 391-402, 2002.

[29] D. B. Williams and C. B. Carter, Transmission Electron Mircroscopy, New York: Springer, 2009.

[30] S. J. Pennycook and P. D. Nellist, Scanning Transmission Electron Microscopy, New York: Springer, 2011.

[31] I. P. Jones, Chemical Microanalysis Using Electron Beams, London: Institute of Materials, 1992.

[32] C. Kisielowski, P. Specht, S. M. Gygax, B. Barton, H. A. Calderon, J. H. Kang and R. Cieslinski, "Instrumental requirements for the detection of electron beam-induced object excitations at the single atom level in high-resolution transmission electron microscopy," Micron, 2014.

[33] W. R. Tyson and W. A. Miller, "Surface Free Energies of Solid Metals: Estimation from Liquid Surface Tension Measurements," Surf. Sci., vol. 62, no. 1, pp. 267-276, 1977.

[34] Y. Zhu, Q. M. Ramasse, M. Brorson, P. G. Moses, L. P. Hansen, C. F. Kisielowski and S. Helveg, "Visualizing the Stoichiometry of Industrial-Style Co-Mo-S Catalysts with SingleAtom Sensitivity," Angew. Chem. Int. Edit., vol. 126, 2014.

[35] B. J. Tan, K. J. Klabunde and P. M. A. Sherwood, "XPS Studies of Solvated Metal Atom Dispersed Catalysts," J. Am. Chem. Soc., vol. 113, pp. 855-861, 1991.

[36] Z.-j. Wang, Z. Yan, C.-j. Liu and D. W. Goodman, "Surface Science Studies on Cobalt FischerTropsch Catalysts," ChemCatChem, vol. 3, no. 3, pp. 551-559, 2011.

[37] G. Prieto, A. Martínez, P. Concepción and R. Moreno-Tost, "Cobalt particle size effects in Fischer-Tropsch synthesis: structural and in situ spectroscopic characterisation on reverse micelle-synthesized Co/ITQ-2 model catalysts," J. Catal., vol. 266, pp. 129-144, 2009. 
[38] J.-S. Girardon, A. S. Lermontov, L. Gengembre, P. A. Chernavskii, A. Griboval-Constant and A. Y. Khodakov, "Effect of cobalt precusor and pretreatment conditions on the structure and catalytic performance of cobalt silica-supported Fischer-Tropsch catalysts," J. Catal., vol. 230, pp. 339-352, 2005.

[39] M. A. Mohamed and S. A. Halway, "Kinetic and mechanistic study of the non-isothermal decomposition of manganese(II) acetate tetrahydrate," Thermochim. Atca., vol. 242, pp. 173-186, 1994.

[40] C. L. Pieck and J. M. Parera, "Differential Effect of Coke Burning with Oxygen or Ozone on Pt-Re Interaction in Pt-Re/Al2O3," Stud. Surf. Sci. Catal., vol. 111, pp. 433-438, 1997.

[41] G. Blanchard, H. Charcosset, M. T. Chenebaux and M. Primet, "Preparation of Amumina or Silica Supported Platinum-Ruthenium Bimetallic Catalysts," Stud. Surf. Sci. Catal., vol. 3, pp. 197-210, 1979.

[42] J. P. den Breejen, A. M. Frey, J. Yang, A. Holmen, M. M. van Schooneveld, F. M. F. de Groot, O. Stephan, J. H. Bitter and K. P. de Jong, "A Highly Active and Selectivity Manganese Oxide Promoted Cobalt-on-Silica Fischer-Tropsch Catalyst," Top. Catal., vol. 54, pp. 768-777, 2011.

[43] T. E. Feltes, Y. Zhao, R. F. Klie, R. J. Meyer and J. R. Regalbuto, "The Influence of Preparation Method on Mn-Co Interactions in Mn/Co/TiO2 Fischer-Tropsch Catalysts," ChemCatChem, vol. 2, pp. 1065-1068, 2010.

[44] G. L. Bezemer, J. H. Bitter, H. P. Kuipers, H. Oosterbeek, J. E. Holewijn, X. Xu, F. Kapteijn, A. J. van Dillen and K. P. de Jong, "Cobalt Particle Size Effects in the Fischer-Tropsch Reaction Studied with Carbon Nanofiber Supported Catalysts," J. Am. Chem. Soc., vol. 128, no. 12, pp. 3956-3964, 2006. 
Table 1. Correlation metrics for simulated images of Co-Mn nanoparticles with different levels of segregation. Representative images of the three patterns are given in Fig. 5A. An explanation of each metric is given in the experimental section.

\begin{tabular}{lllll}
\hline Pattern & PCC & SRCC & MCC & SSIM \\
\hline Alloying & 0.99 & 1.00 & 1.00 & 0.99 \\
Surface Associated & 0.75 & 0.73 & 0.81 & 0.78 \\
Segregated & 0.03 & 0.02 & 0.18 & 0.27 \\
\hline
\end{tabular}


Table 2. Correlation metrics for Co-Mn catalysts with different pretreatment conditions. The initially calcined catalyst was calcined in air at $673 \mathrm{~K}$ and then reduced in $\mathrm{H}_{2}$ at $673 \mathrm{~K}$. The directly reduced catalyst was reduced in $\mathrm{H}_{2}$ at $673 \mathrm{~K}$. An explanation of each metric is given in the experimental section.

\begin{tabular}{lllll}
\hline Sample & PCC & SRCC & MCC & SSIM \\
\hline Initially Calcined & 0.28 & 0.34 & 0.99 & 0.26 \\
Directly Reduced & 0.74 & 0.78 & 1.00 & 0.56 \\
\hline
\end{tabular}


A)

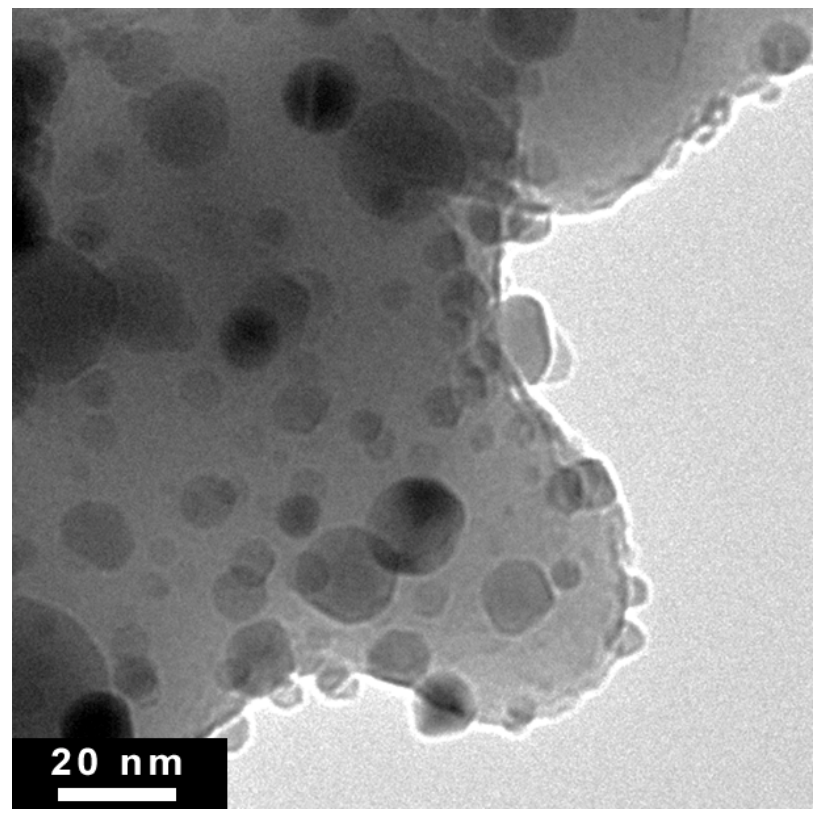

B)

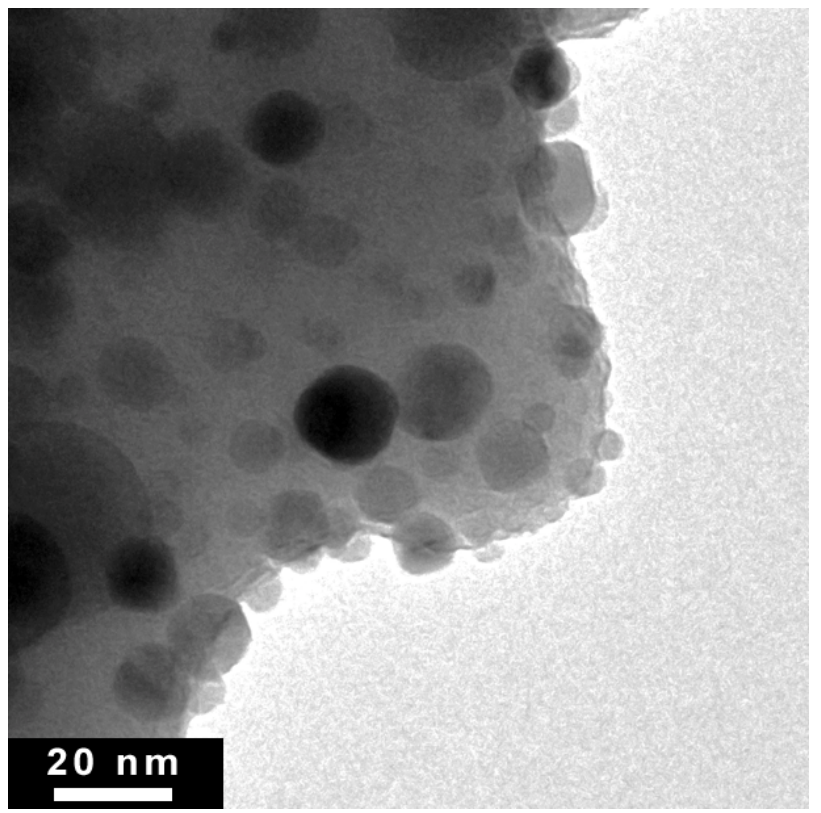

Figure 1. Bright-field TEM images of Co (left) and Co-Mn (Mn/Co $=0.1$, right) catalysts. Images were acquired using an FEI Tecnai F12 at 120kV. Although metal nanoparticles can be identified against the silica support material, it is not possible to distinguish unambiguously between Co and $\mathrm{Mn}$. 
B)
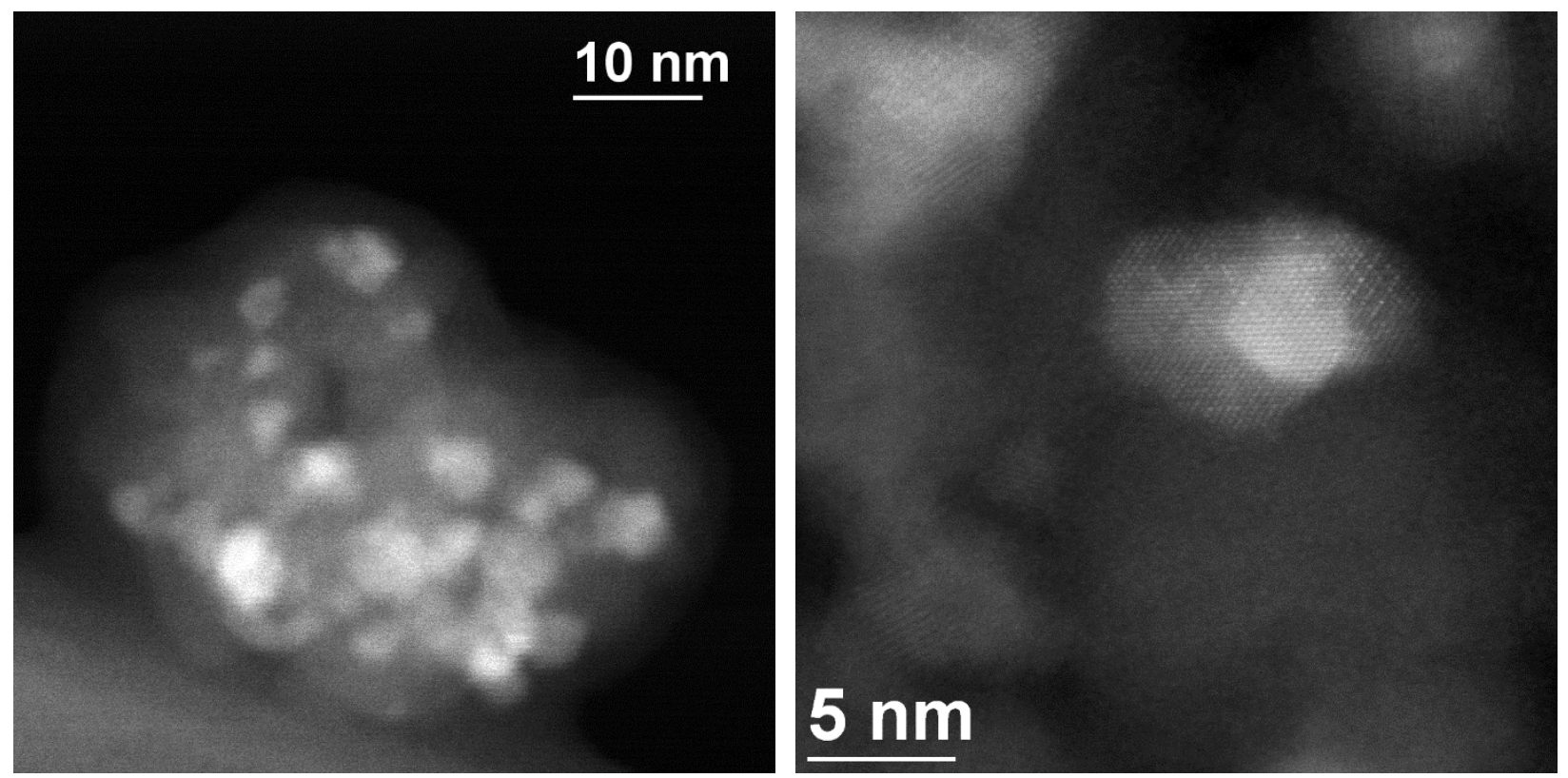

Figure 2. HAADF-STEM images of a Co-Mn catalyst sample $(\mathrm{Mn} / \mathrm{Co}=0.1)$ taken in a FEI Tecnai T20 (A) and the TEAM I (B), both at 80kV. It is not obvious where the Co and $\mathrm{Mn}$ are located with respect to each other even upon examining the lattice of the metal nanoparticles. 
A)
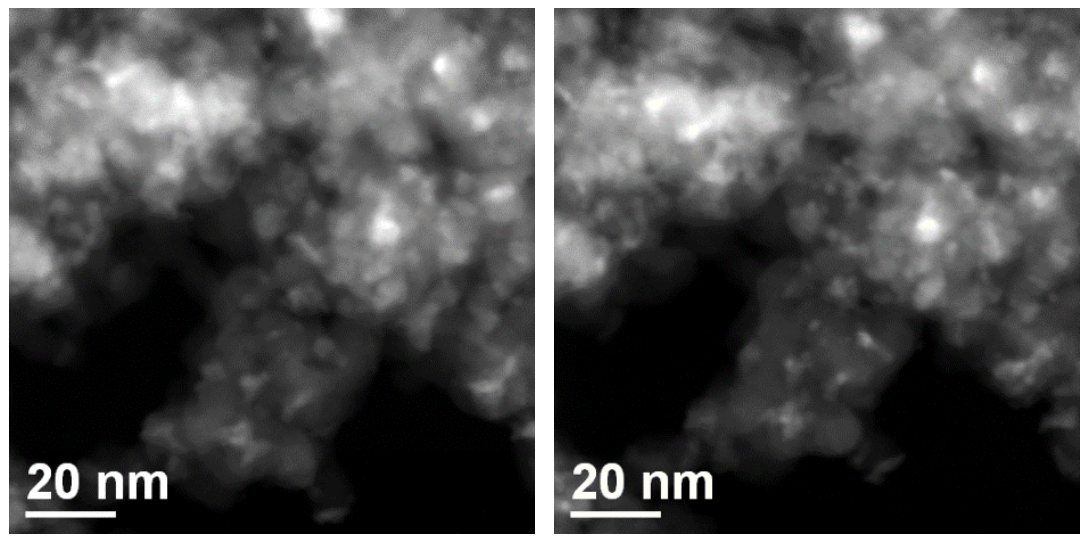

C)

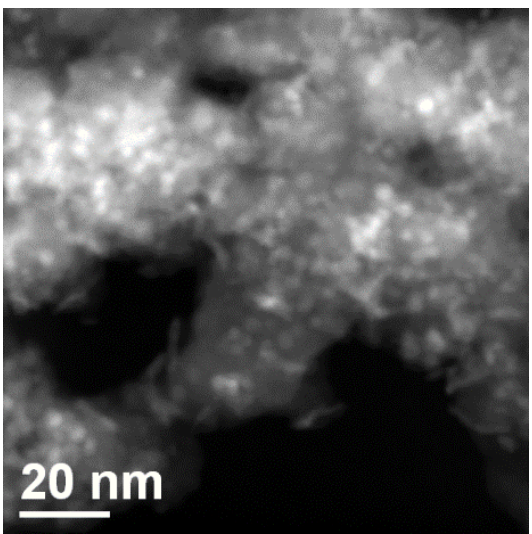

Figure 3. Accumulation of damage to a Mn-promoted Co catalyst $(\mathrm{Mn} / \mathrm{Co}=0.1)$ by extended rastering with the electron beam in STEM mode. Images were acquired in succession at $200 \mathrm{kV}$, $0.6 \mathrm{nA}$ beam current, and $50 \mu \mathrm{s}$ dwell time. A) initial catalyst image; B) catalyst image after 8 min (typical mapping time); C) catalyst image after $45 \mathrm{~min}$. Comparisons of accelerating voltage, beam current, and dwell time are included in Supporting Information SI3. 
A)

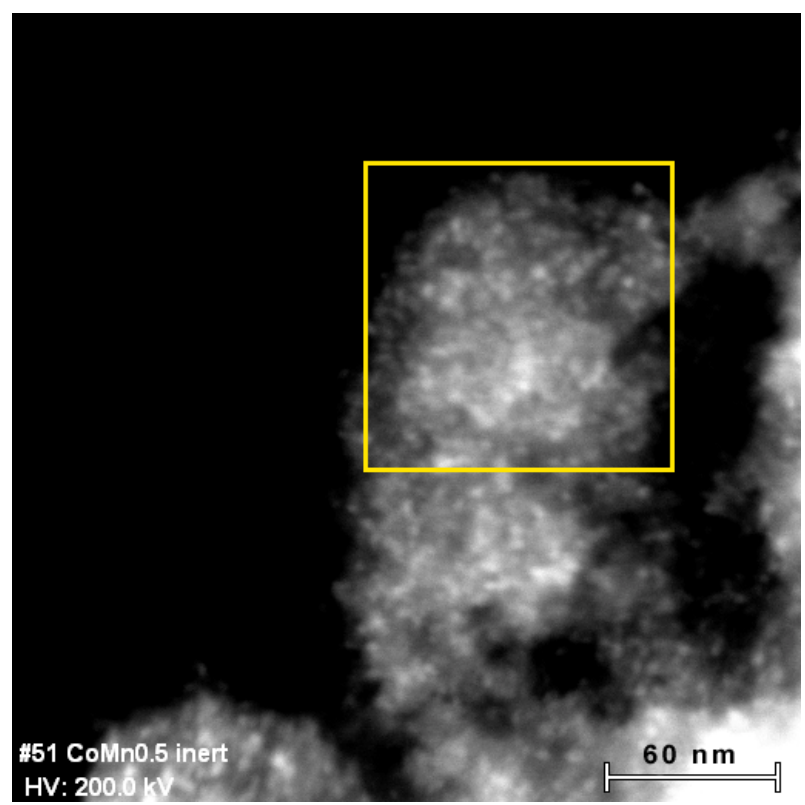

C)

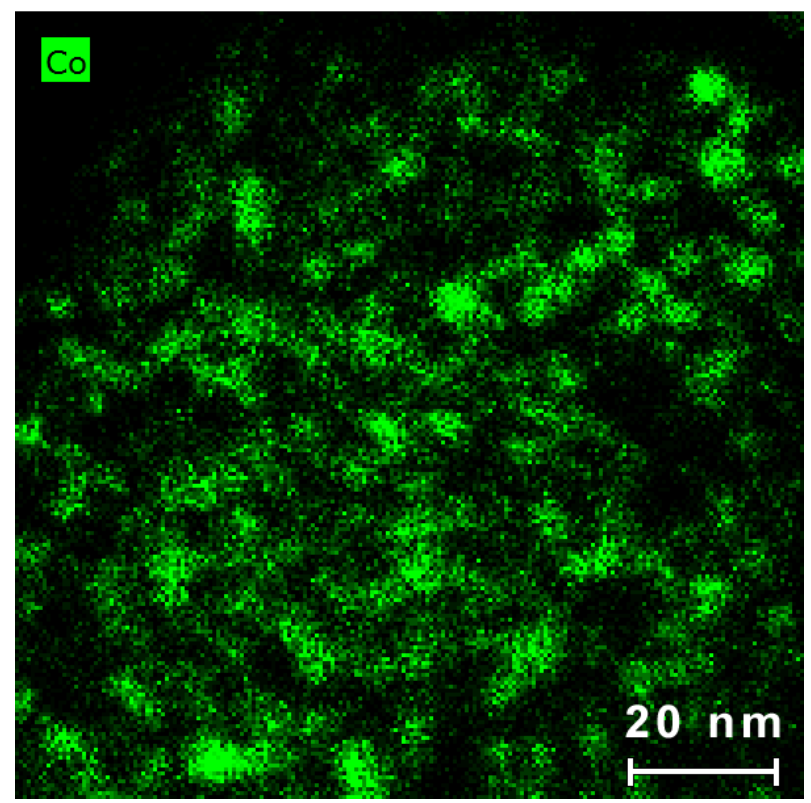

B)

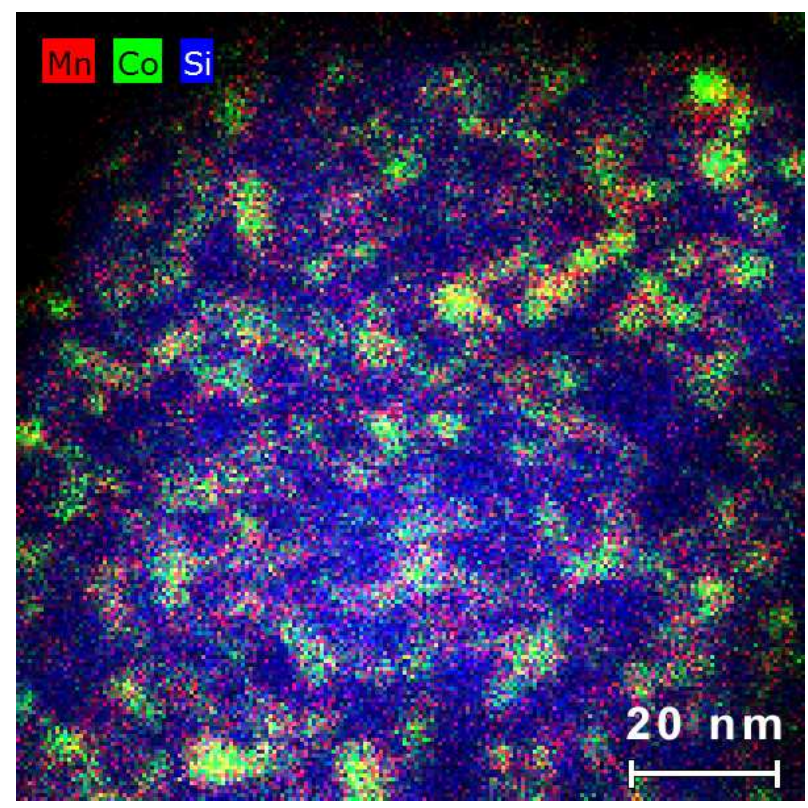

D)

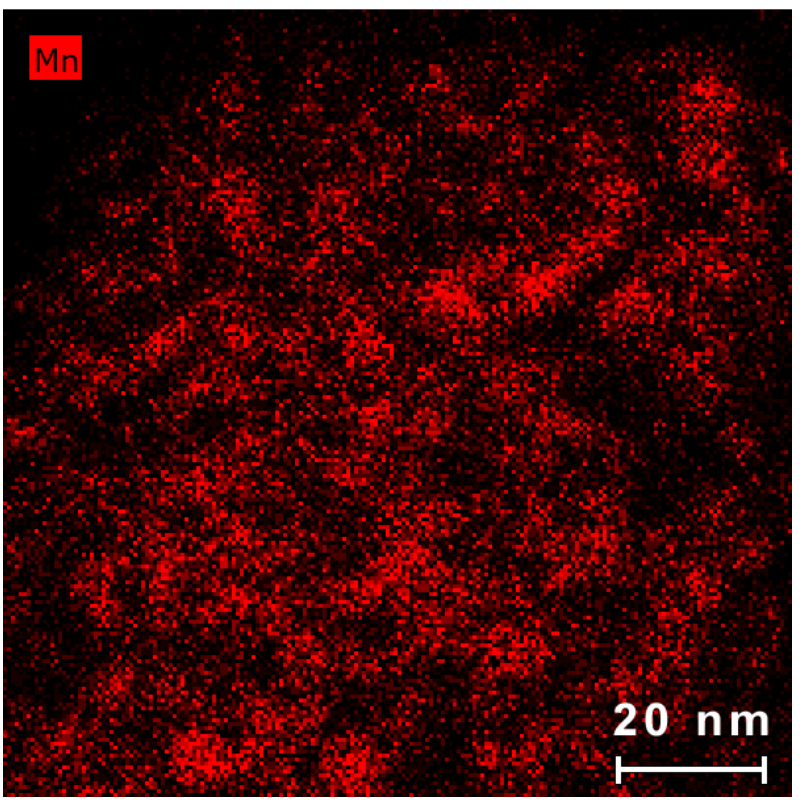

Figure 4. Elemental map of a Co-Mn catalyst (directly reduced, $\mathrm{Mn} / \mathrm{Co}=0.5$ ) obtained by STEM-EDS. A) HAADF image of catalyst particle with boxed region indicating where the elemental map was acquired; $B$ ) STEM-EDS elemental map; $C$ ) Co channel; $D$ ) Mn channel. Images were acquired at $200 \mathrm{kV}$ accelerating voltage and $0.6 \mathrm{nA}$ beam current. 


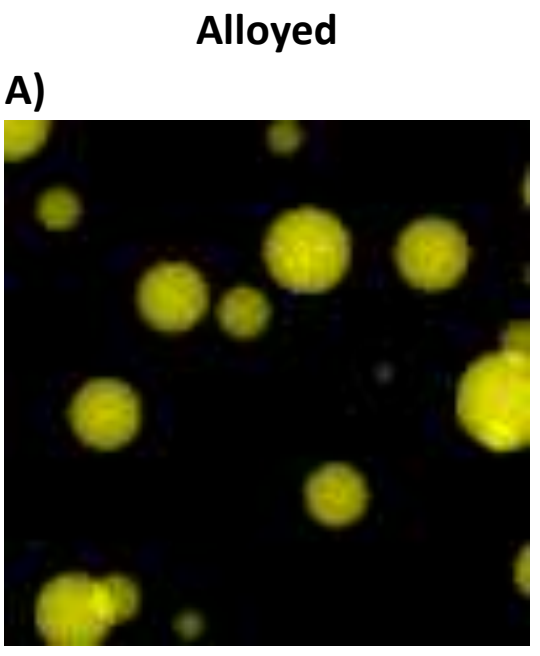

Surface Associated

Segregated

A)
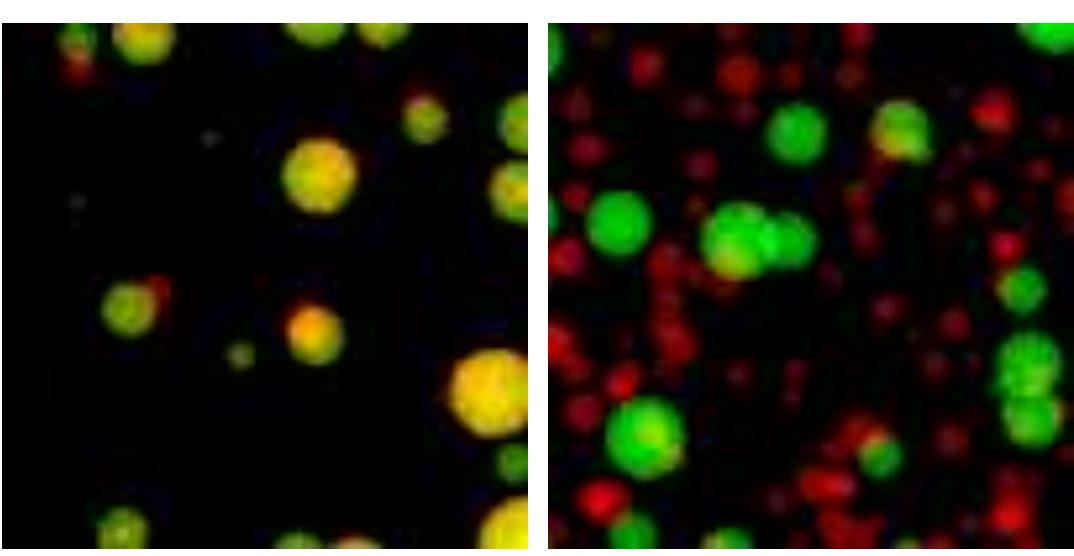

B)
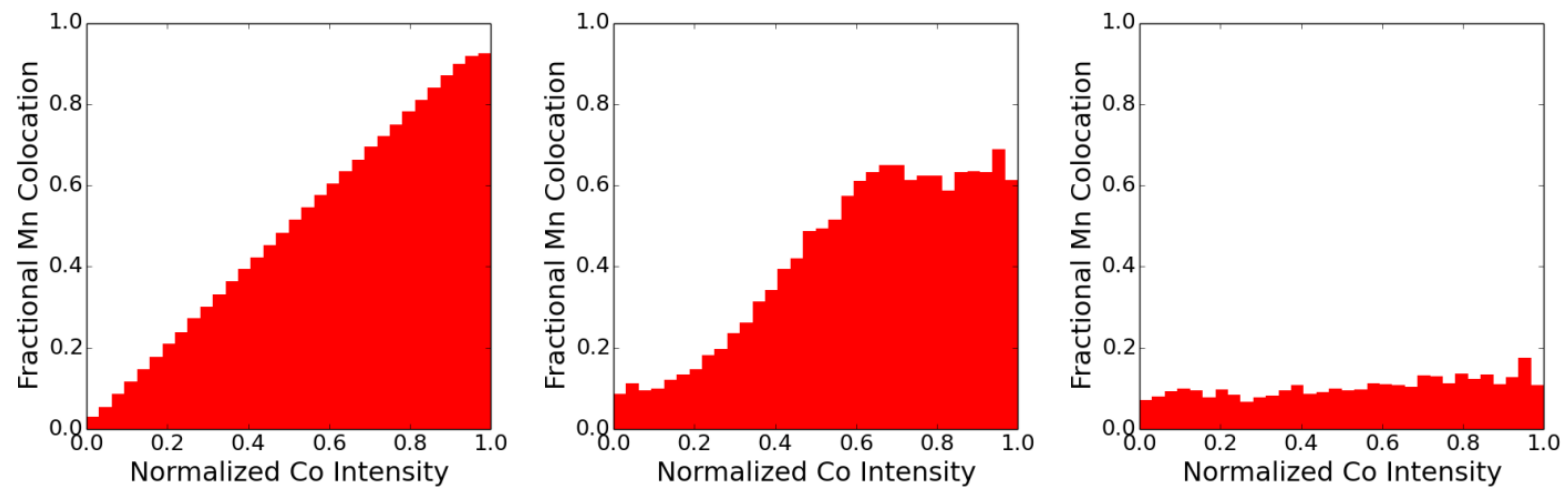

C)
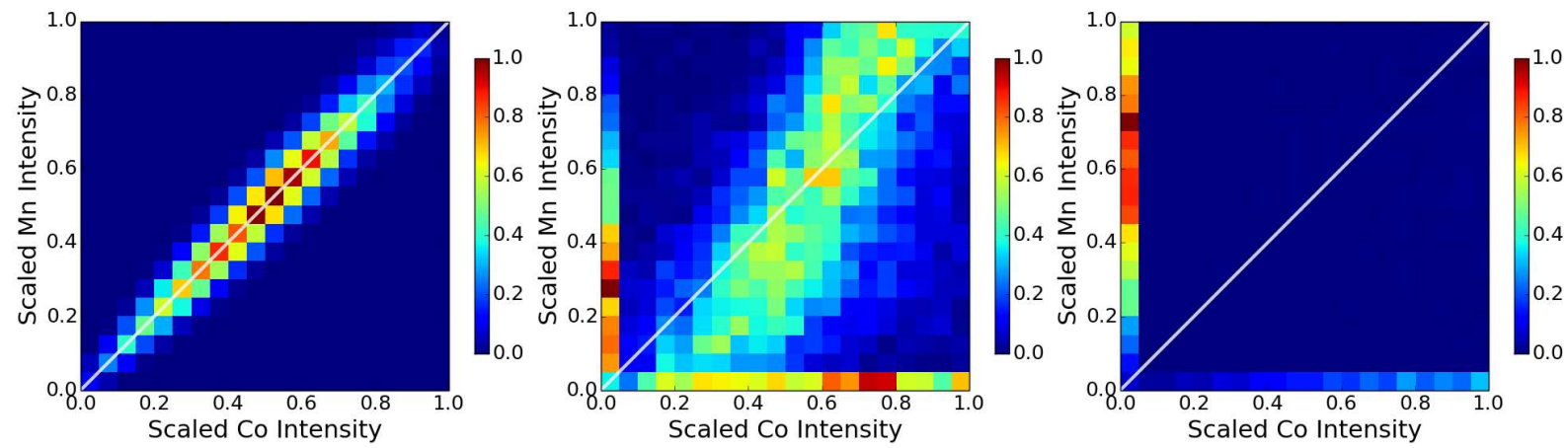
D)

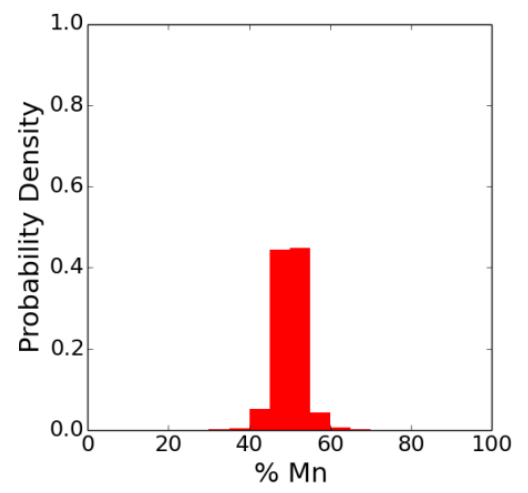

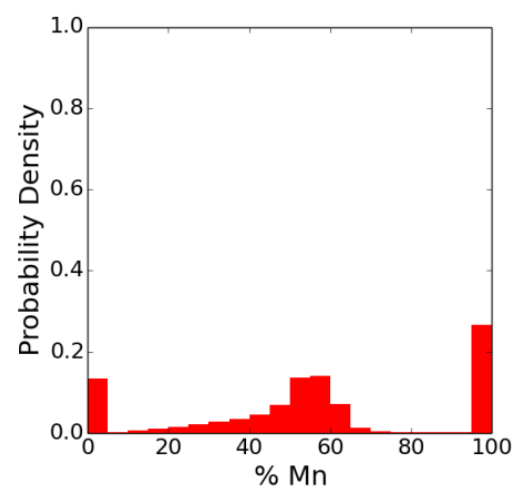

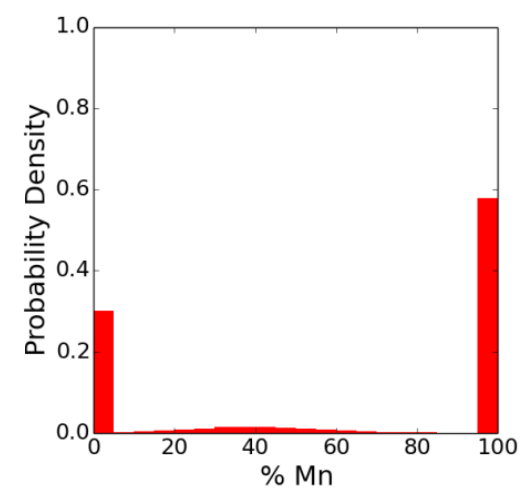

Figure 5. Simulated images and correlation analysis plots of Co-Mn nanoparticles with different levels of element segregation. Left column: alloying; middle column: surface association; right column: complete segregation. A) representative simulated images depicting different extends of element association; B) colocation probability plots; C) 2D histograms; D) composition histograms. 
Initially Calcined

A)

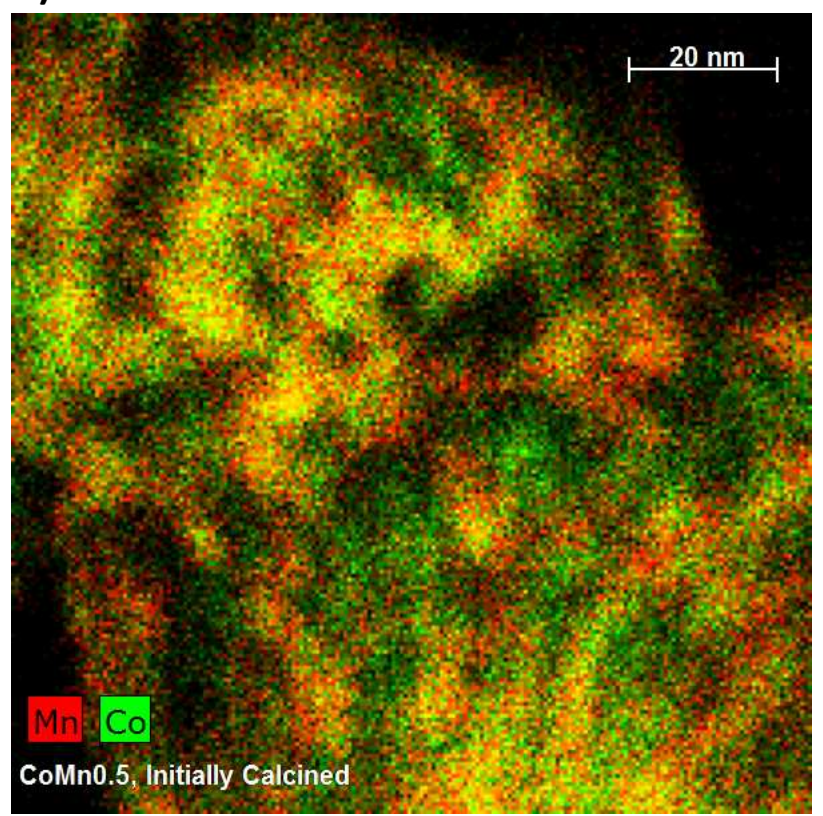

B)

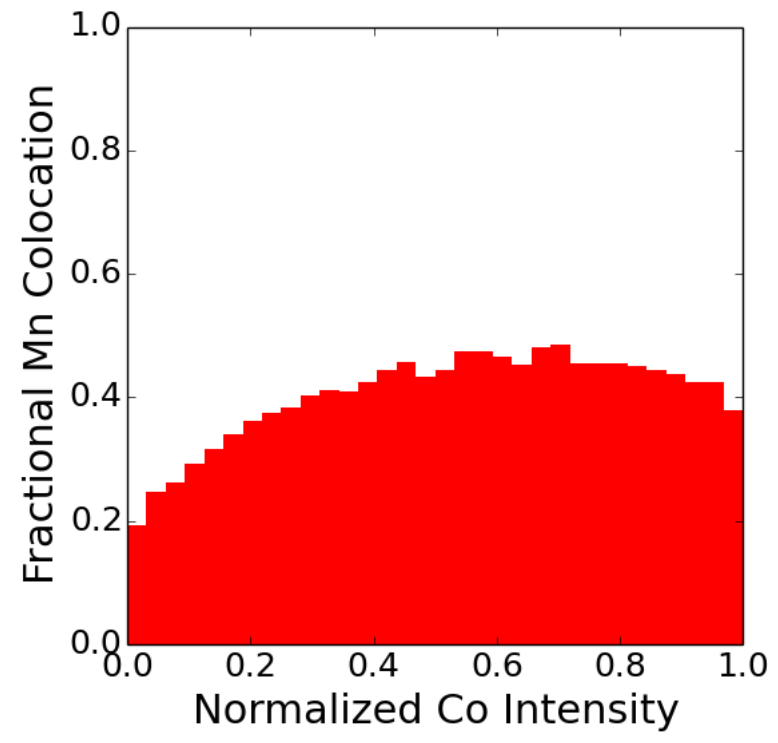

Directly Reduced
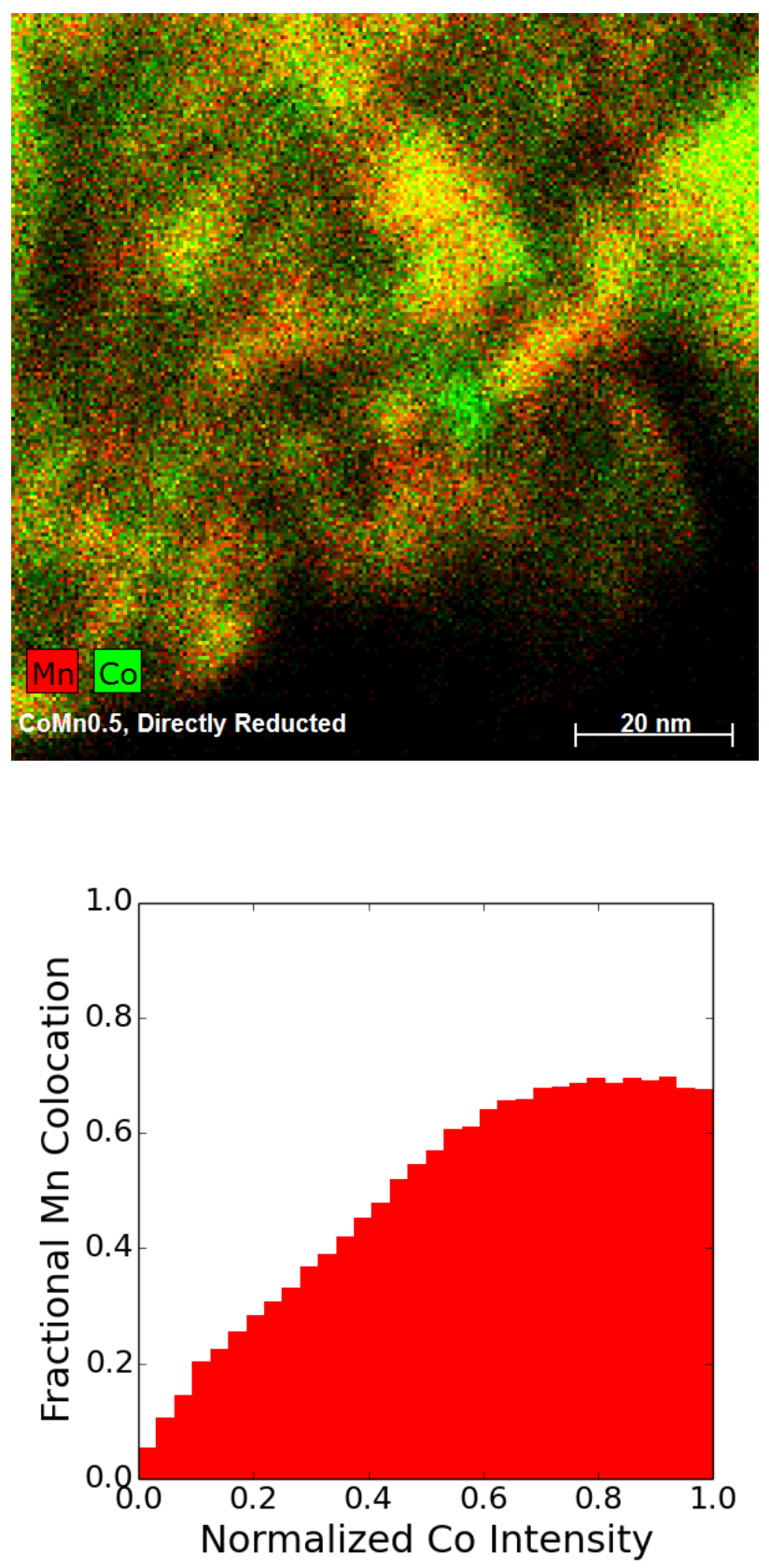

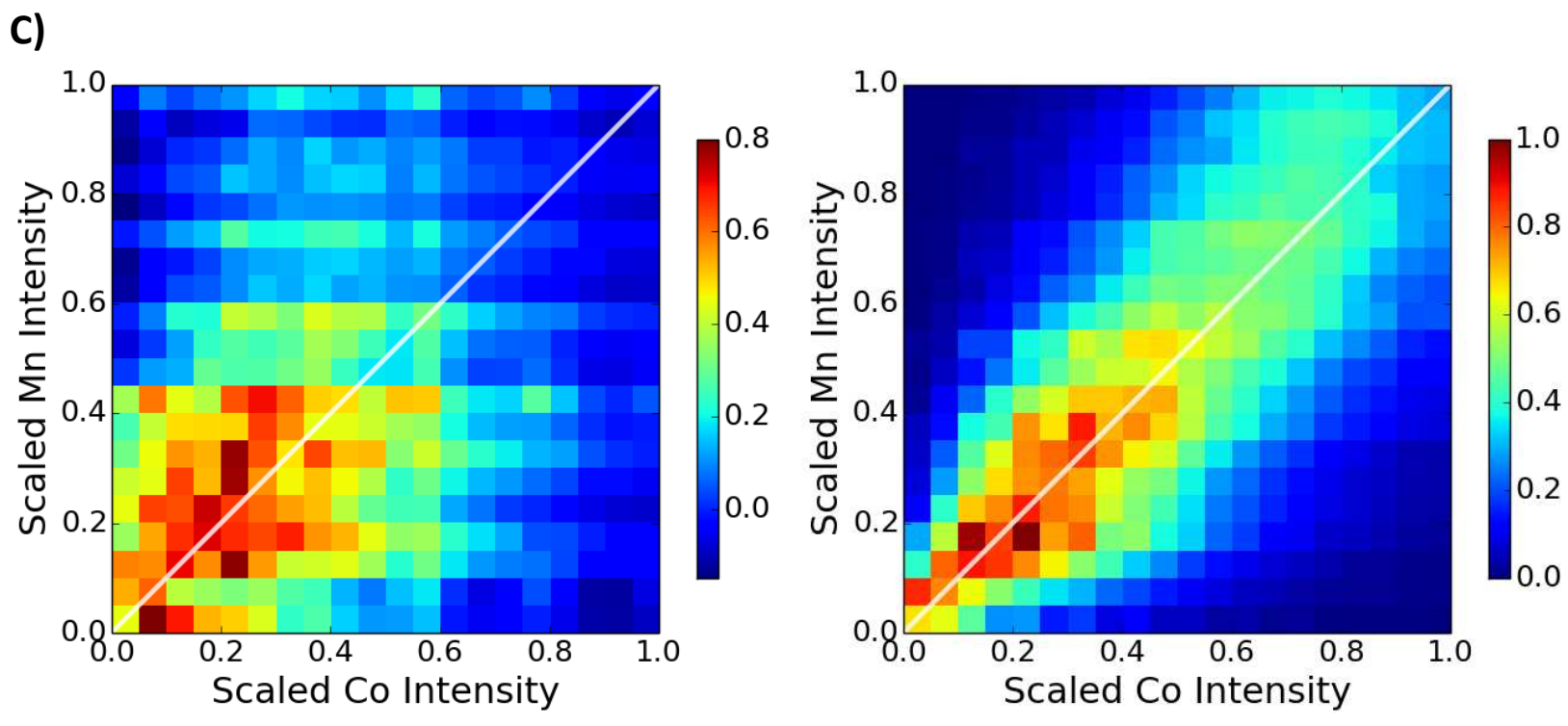

D)
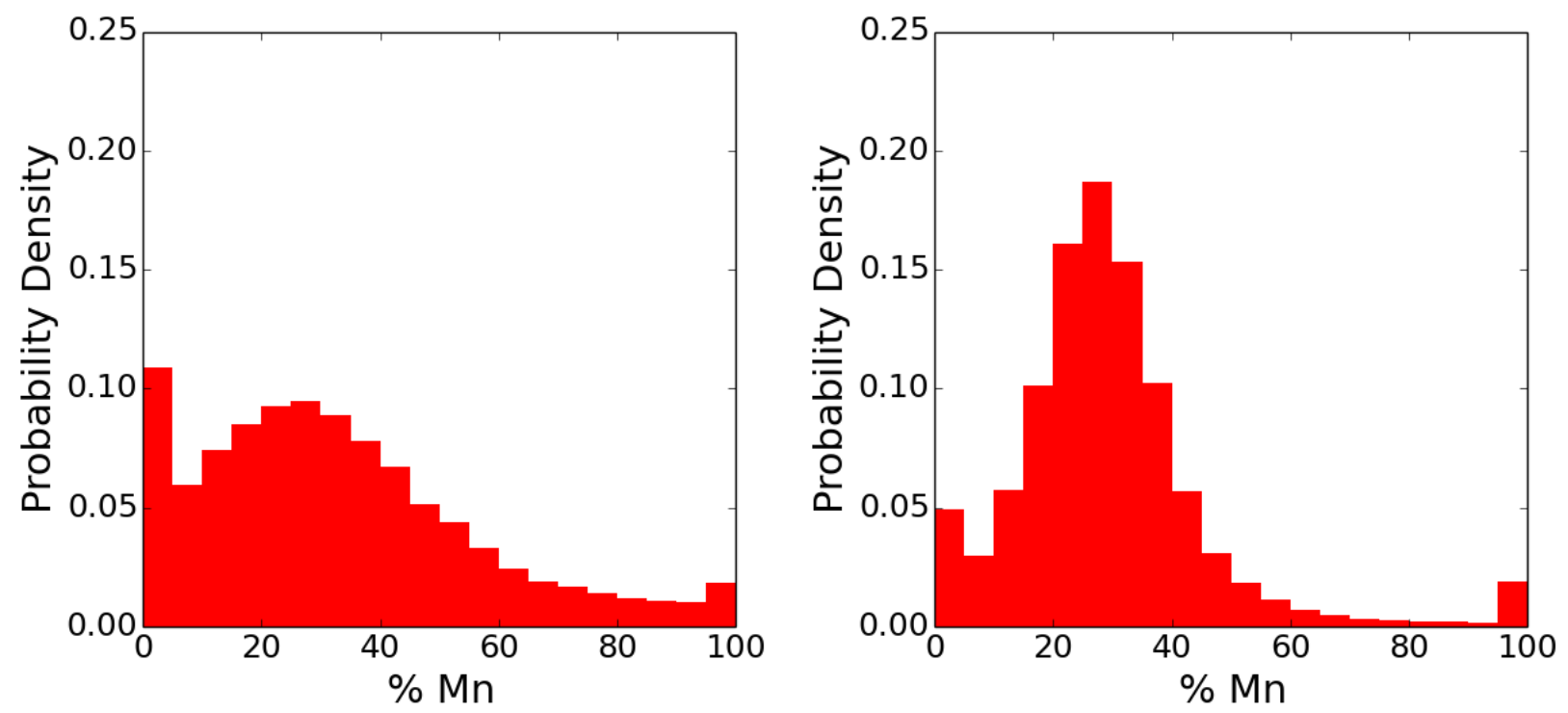

Figure 6. STEM-EDS elemental maps and correlation analysis plots of Co-Mn catalysts ( $\mathrm{Mn} / \mathrm{Co}=$ 0.5 ) with different pretreatment conditions. Left column: catalyst was initially calcined at $673 \mathrm{~K}$ and then reduced at $673 \mathrm{~K}$; right column: catalyst was directly reduced at $673 \mathrm{~K}$. A) representative elemental maps; B) colocation probability plots; C) $2 \mathrm{D}$ histograms; $D$ ) composition histograms. 

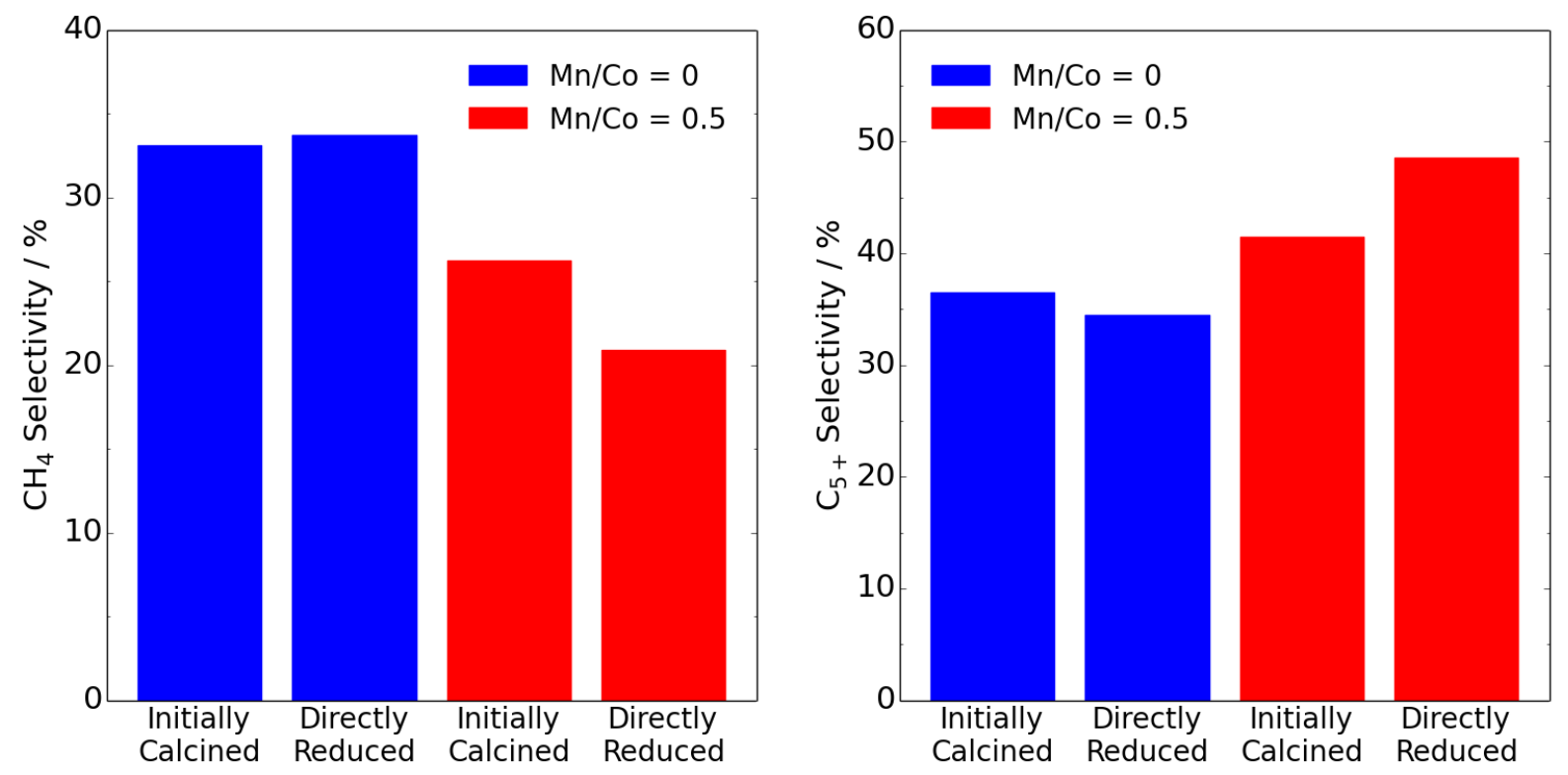

Figure 7. Methane (left) and $\mathrm{C}_{5+}$ hydrocarbon (right) selectivities of the Co and Co- $\mathrm{Mn}$ ( $\mathrm{Mn} / \mathrm{Co}$ $=0.5)$ catalysts under Fischer-Tropsch synthesis. Measurements were taken at $1 \mathrm{bar}, 493 \mathrm{~K}$, and $\mathrm{H}_{2} / \mathrm{CO}=2$, and the data were extrapolated to $0 \% \mathrm{CO}$ conversion. The plotted data is product selectivity on a molar carbon basis. 